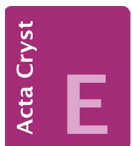

CRYSTALLOGRAPHIC COMMUNICATIONS

ISSN 2056-9890

Received 8 May 2015

Accepted 13 May 2015

Edited by A. Van der Lee, Université de Montpellier II, France

\section{Crystal structure of pentapotassium dihydrogen nonavanadato(V)platinate(IV) nonahydrate}

\author{
Hea-Chung Joo and Uk Lee*
}

Department of Chemistry, Pukyong National University, 599-1 Daeyeon 3-dong, Nam-gu, Busan 608-737, Republic of Korea. *Correspondence e-mail: uklee@pknu.ac.kr

The title compound, $\mathrm{K}_{5}\left[\mathrm{H}_{2} \mathrm{PtV}_{9} \mathrm{O}_{28}\right] \cdot 9 \mathrm{H}_{2} \mathrm{O}$, containing the nonavanadoplatinate(IV) polyanion, was obtained by hydrothermal reaction at $\mathrm{pH}=4.2$. The polyanion has approximate $m m 2\left(C_{2 v}\right)$ symmetry. The two platinum-bound $\mu_{2}-\mathrm{O}$ atoms are protonated in the polyanion. The heteropolyanions form inversion-generated dimers, $\left\{\left[\mathrm{H}_{2} \mathrm{PtV}_{9} \mathrm{O}_{28}\right]_{2}\right\}^{10-}$, held together by $\mu_{2}-\mathrm{O}-\mathrm{H} \cdots \mu_{2}-$ $\mathrm{O}$ and $\mu_{2}-\mathrm{O}-\mathrm{H} \cdots \mu_{3}-\mathrm{O}$ hydrogen bonds. All $\mathrm{K}^{+}$cations are located on general positions of the space group $P \overline{1}$.

\section{Structural commentary}

vanadoplatinate(IV) polyanion; heteropolyanions; hydrogen bonding

CCDC reference: 1400654

Supporting information: this article has supporting information at journals.iucr.org/e

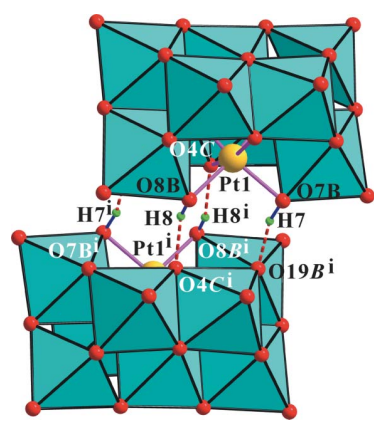

OPEN $\odot$ ACCESS
Two heteropolyanions that belong to the decavanadate structure system (Lee, 2006) have recently been reported: the tellurium derivative $\left[\mathrm{H}_{n} \mathrm{TeV}_{9} \mathrm{O}_{28}\right]^{(5-n)}(n=1$ and 2$)$, described by Konaka et al. (2011), and the platinum heteropolyoxidovanadate, $\left[\mathrm{H}_{2} \mathrm{PtV}_{9} \mathrm{O}_{28}\right]^{5-}$, reported by our group in the form of its sodium salt, $\mathrm{Na}_{5}\left[\mathrm{H}_{2} \mathrm{PtV}_{9} \mathrm{O}_{28}\right] \cdot 21 \mathrm{H}_{2} \mathrm{O}$ (Lee et al., 2008) and a guanidinium salt, $\left(\mathrm{CH}_{6} \mathrm{~N}_{3}\right)_{5}\left[\mathrm{H}_{2} \mathrm{PtV}_{9} \mathrm{O}_{28}\right]$ (Joo et al., 2011). The Te heteroatom of the $\left[\mathrm{H}_{n} \mathrm{TeV}_{9} \mathrm{O}_{28}\right]^{(5-n)}$ polyanion was located on two sites (corresponding to the Pt1 and V4 sites in the title compound) by disorder. However, the Pt atom does not show any disorder in three $\left[\mathrm{H}_{2} \mathrm{PtV}_{9} \mathrm{O}_{28}\right]^{5-}$ polyanions. We herein report the structure of the title compound because it could contribute to our knowledge of the structural characteristics of the $\left[\mathrm{H}_{2} \mathrm{PtV}_{9} \mathrm{O}_{28}\right]^{5-}$ polyanion.

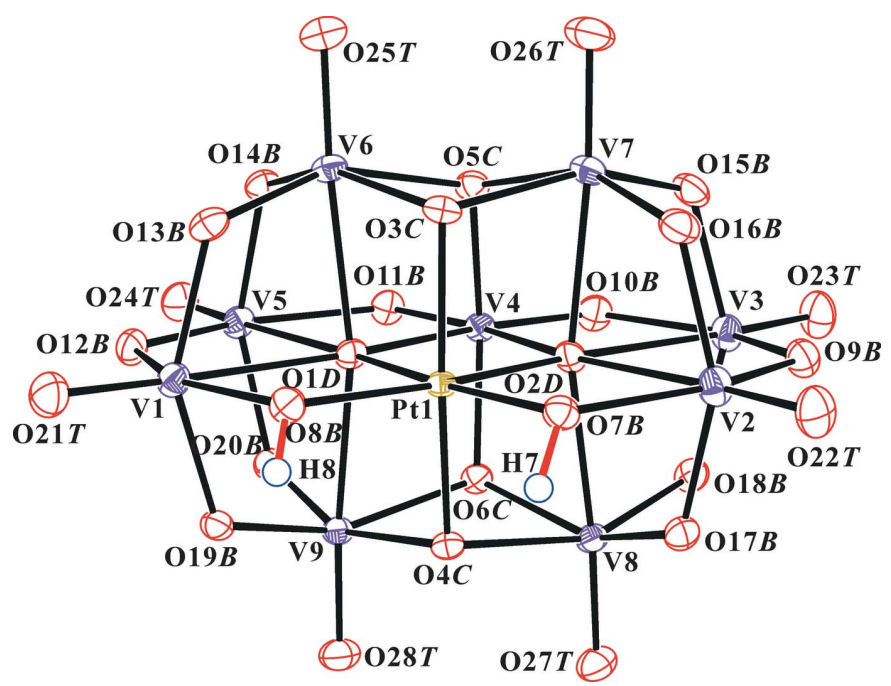

Figure 1

The molecular structure of the heteropolyanion in the title compound showing the atom-numbering scheme. Displacement ellipsoids are drawn at the $50 \%$ probability level. $\mathrm{H}$ atoms are presented as small spheres of arbitrary radius. 


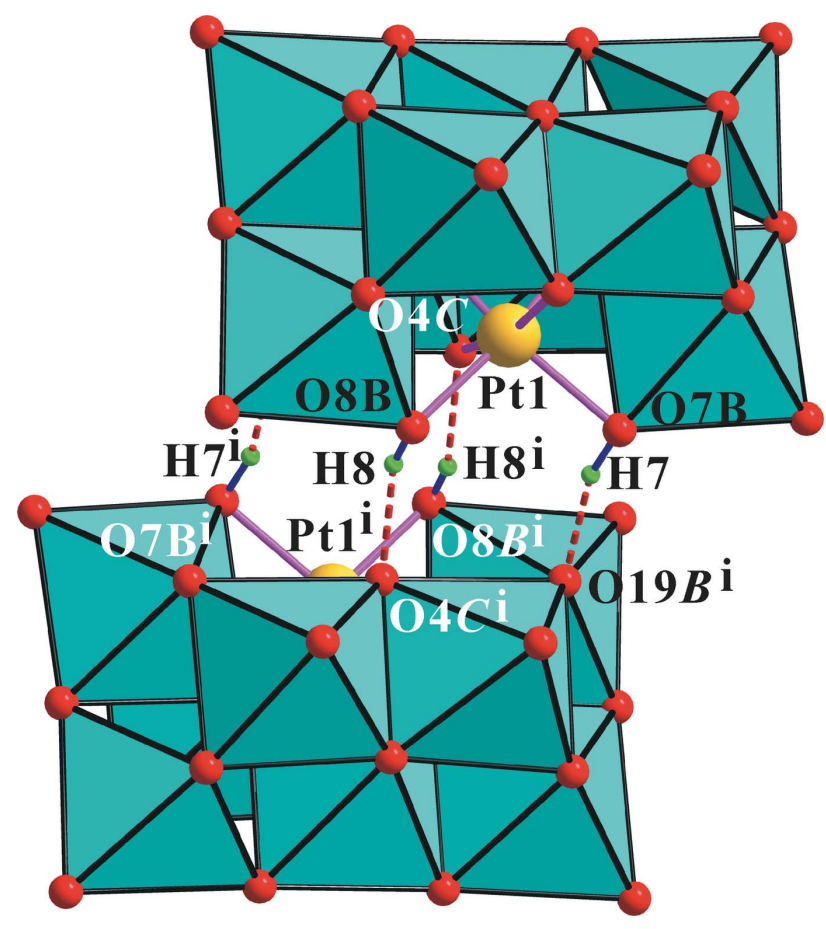

Figure 2

Polyhedral view of the inter-anion hydrogen bonds (dotted lines) in the crystal structure of the title compound. [Symmetry code: (i) $-x,-y+1$, $-z$.

Fig. 1 shows the structure of the heteropolyanion in the title compound. The $\mathrm{O}$ atoms of the clusters were designated as $\mathrm{O} T$ (terminal, $\mathrm{V}=\mathrm{O}), \mathrm{O} B$ (bridging, $\left.\mu_{2}-\mathrm{O}\right), \mathrm{O} C\left(\mu_{3}-\mathrm{O}\right)$, and $\mathrm{O} D\left(\mu_{4}-\mathrm{O}\right)$. All atoms in the polyanions are located in general positions. The protonated $\mathrm{O} B$ atoms in the polyanions were identified by the locations in the difference Fourier maps of the $\mathrm{H}$ atoms bound to atoms $\mathrm{O} 7 \mathrm{~B}$ and $\mathrm{O} 8 \mathrm{~B}$ and local structural features, as seen previously in sodium and guanidinium salts, respectively. The geometry of the anion agrees well with that in sodium and guanidinium salts. The nine $\left[\mathrm{VO}_{6}\right]$ octahedra in the polyanion are distorted [range of $\mathrm{V}-\mathrm{O}$ distances $=1.596(3)-2.403(3) \AA]$, while the $\left[\mathrm{PtO}_{6}\right]$ octahedron is relatively regular $[\mathrm{Pt}-\mathrm{O}=1.985(3)-2.036(3) \AA]$. The two platinum bound $\mu_{2}-\mathrm{O}$ atoms are protonated in the polyanion. These protons are particularly important in the solid state as they lead to the formation of a dimeric assembly, $\left\{\left[\mathrm{H}_{2} \mathrm{PtV}_{9} \mathrm{O}_{28}\right]_{2}\right\}^{10-}$, through each of the two $\mu_{2}-\mathrm{O} 7 B-\mathrm{H} 7 \cdots \mu_{2^{-}}$ $\mathrm{O} 19 B$ and $\mu_{2}-\mathrm{O} 8 B-\mathrm{H} 8 \cdots \mu_{3}-\mathrm{O} 4 C$ interanion hydrogen bonds (Fig. 2 and Table 1).

The $\mathrm{K}^{+}$ions are variously coordinated by $\mathrm{O}$ atoms as $\left[\mathrm{K} 1(\mathrm{OB})(\mathrm{OT})_{2}(\mathrm{OW})_{5}\right]^{+}$in the range $2.725(5)-3.351(6) \AA$, $\left[\mathrm{K} 2(\mathrm{OB})_{2}(\mathrm{OT})_{3}(\mathrm{OW})_{3}\right]^{+}$in the range $2.722(4)-3.156(5) \AA$, $\left[\mathrm{K} 3(\mathrm{OB})(\mathrm{OT})_{4}(\mathrm{OW})_{4}\right]^{+}$in the range $2.844(4)-3.151(3) \AA$, $\left[\mathrm{K} 4(\mathrm{OB})(\mathrm{OT})_{2}(\mathrm{OW})_{4}\right]^{+}$in the range $2.733(5)-3.284(7) \AA$, and $\left[\mathrm{K} 5(\mathrm{OB})_{2}(\mathrm{OT})_{2}(\mathrm{OW})_{3}\right]^{+}$in the range $2.734(6)$ 2.996 (4) А. The bond-valence sums (BVS; Brown \& Altermatt, 1985; Brese \& O'Keeffe, 1991) for the K1, K2, K3, K4, and $\mathrm{K} 5$ cations are $0.99,1.12,1.04,0.81$, and 1.10 v.u, respectively (total v.u. $=5.06$ ).
Table 1

Hydrogen-bond geometry $\left(\AA,^{\circ}\right)$.

\begin{tabular}{|c|c|c|c|c|}
\hline$D-\mathrm{H} \cdots A$ & $D-\mathrm{H}$ & $\mathrm{H} \cdots A$ & $D \cdots A$ & $D-\mathrm{H} \cdots A$ \\
\hline $\mathrm{O} 7 B-\mathrm{H} 7 \cdots \mathrm{O} 19 B^{\mathrm{i}}$ & $0.90(8)$ & $1.86(8)$ & $2.738(4)$ & $164(8)$ \\
\hline $\mathrm{O} 8 B-\mathrm{H} 8 \cdots \mathrm{O} 4 C^{\mathrm{i}}$ & $0.77(6)$ & $1.90(6)$ & $2.645(5)$ & $161(6)$ \\
\hline $\mathrm{O} 1 W-\mathrm{H} 1 B \cdots \mathrm{O} 9 B$ & $0.83(3)$ & $2.50(6)$ & $3.127(6)$ & $133(7)$ \\
\hline $\mathrm{O} 1 W-\mathrm{H} 1 A \cdots \mathrm{O} 8 W$ & $0.84(3)$ & $2.10(3)$ & $2.930(9)$ & $170(8)$ \\
\hline $\mathrm{O} 2 W-\mathrm{H} 2 A \cdots \mathrm{O} 15 B$ & $0.87(3)$ & $1.88(3)$ & $2.728(6)$ & $166(8)$ \\
\hline $\mathrm{O} 2 W-\mathrm{H} 2 B \cdots \mathrm{O} 11 B^{\mathrm{ii}}$ & $0.86(3)$ & $2.23(5)$ & $2.975(6)$ & $145(8)$ \\
\hline $\mathrm{O} 3 W-\mathrm{H} 3 A \cdots \mathrm{O} 3 C^{\mathrm{iii}}$ & $0.84(3)$ & $1.83(3)$ & $2.673(5)$ & $177(10)$ \\
\hline $\mathrm{O} 3 W-\mathrm{H} 3 B \cdots \mathrm{O} 7 W$ & $0.83(3)$ & $2.09(5)$ & $2.875(7)$ & $158(8)$ \\
\hline $\mathrm{O} 4 W-\mathrm{H} 4 A \cdots \mathrm{O} 13 B^{\mathrm{i}}$ & $0.83(3)$ & $1.88(3)$ & $2.680(5)$ & $162(7)$ \\
\hline $\mathrm{O} 4 W-\mathrm{H} 4 B \cdots \mathrm{O} 14 B^{\mathrm{iv}}$ & $0.83(3)$ & $2.10(4)$ & $2.850(5)$ & $151(7)$ \\
\hline $\mathrm{O} 5 W-\mathrm{H} 5 A \cdots \mathrm{O} 2 W^{\mathrm{v}}$ & $0.87(3)$ & $1.84(3)$ & $2.710(8)$ & $175(8)$ \\
\hline $\mathrm{O} 5 W-\mathrm{H} 5 B \cdots \mathrm{O} 24 T^{\mathrm{vi}}$ & $0.84(3)$ & $2.26(5)$ & $2.972(6)$ & $142(7)$ \\
\hline $\mathrm{O} 6 W-\mathrm{H} 6 A \cdots \mathrm{O} 5 C^{\mathrm{iv}}$ & 0.97 & 1.81 & $2.755(5)$ & 163 \\
\hline $\mathrm{O} 6 W-\mathrm{H} 6 B \cdots \mathrm{O} 10 B^{\mathrm{v}}$ & 0.97 & 2.04 & $2.755(5)$ & 129 \\
\hline $\mathrm{O} 7 W-\mathrm{H} 7 A \cdots \mathrm{O} 7 B^{\mathrm{iii}}$ & $0.85(3)$ & $2.07(4)$ & $2.891(5)$ & $163(8)$ \\
\hline $\mathrm{O} 7 W-\mathrm{H} 7 B \cdots \mathrm{O} 6 C^{\mathrm{v}}$ & $0.84(3)$ & $2.27(6)$ & $2.885(5)$ & $130(6)$ \\
\hline $\mathrm{O} 8 W-\mathrm{H} 8 A \cdots \mathrm{O} 5 W^{\mathrm{V}}$ & $0.86(3)$ & $1.98(5)$ & $2.795(7)$ & $159(10)$ \\
\hline $\mathrm{O} 8 W-\mathrm{H} 8 B \cdots \mathrm{O} 19 B^{\mathrm{vii}}$ & $0.86(3)$ & $2.22(4)$ & $3.031(6)$ & $156(8)$ \\
\hline
\end{tabular}

The polyanion dimers are three-dimensionally linked via $\mathrm{K} \cdots \mathrm{O} T$ and $\mathrm{K} \cdots \mathrm{O} B$ interactions. All water molecules form hydrogen bonds with polyanions except for the $\mathrm{O} 9 \mathrm{~W}$ water molecule (Table 1).

Table 2

Experimental details.

\begin{tabular}{|c|c|}
\hline \multicolumn{2}{|l|}{ Crystal data } \\
\hline Chemical formula & $\mathrm{K}_{5}\left[\mathrm{H}_{2} \mathrm{PtV}_{9} \mathrm{O}_{28}\right] \cdot 9 \mathrm{H}_{2} \mathrm{O}$ \\
\hline$M_{\mathrm{r}}$ & 1461.21 \\
\hline Crystal system, space group & Triclinic, $P \overline{1}$ \\
\hline Temperature (K) & 298 \\
\hline$a, b, c(\AA)$ & $10.1663(7), 12.8350(7), 13.615(2)$ \\
\hline$\alpha, \beta, \gamma\left({ }^{\circ}\right)$ & $103.734(5), 106.193(6), 92.480(4)$ \\
\hline$V\left(\AA^{3}\right)$ & $1645.8(3)$ \\
\hline$Z$ & 2 \\
\hline Radiation type & Мo $K \alpha$ \\
\hline$\mu\left(\mathrm{mm}^{-1}\right)$ & 7.42 \\
\hline Crystal size $(\mathrm{mm})$ & $0.21 \times 0.19 \times 0.17$ \\
\hline
\end{tabular}

Data collection

Diffractometer

Absorption correction

$T_{\min }, T_{\max }$

No. of measured, independent and observed $[I>2 \sigma(I)]$ reflections $(\sin \theta / \lambda)_{\max }\left(\AA^{-1}\right)$

Stoe Stadi4
Empirical (using intensity
$\quad$ measurements) (X-SHAPE;
$\quad$ Stoe \& Cie,1996)
$0.301,0.378$
$6797,6797,6242$
0.628

$0.028,0.066,1.10$
6797
526
25
H atoms treated by a mixture of
$\quad$ independent and constrained
$\quad$ refinement
$1.30,-1.46$

$\Delta \rho_{\max }, \Delta \rho_{\min }\left(\mathrm{e} \AA^{-3}\right)$ $1.30,-1.46$

Computer programs: STADI4 and X-RED (Stoe \& Cie, 1996), SHELXS2014 (Sheldrick, 2008), SHELXL2014 (Sheldrick, 2015), ORTEP-3 for Windows (Farrugia, 2012) and DIAMOND (Brandenburg, 1998). 


\section{Synthesis and crystallization}

Single crystals of the title compound were obtained in the same way as the sodium salt reported by Lee et al. (2008) using $\mathrm{K}_{2} \mathrm{Pt}(\mathrm{OH})_{6}$ and $\mathrm{KVO}_{3}$.

\section{Refinement}

Crystal data, data collection and structure refinement details are summarized in Table 2. Atoms $\mathrm{H} 7$ and $\mathrm{H} 8$, bound to $\mu_{2}-\mathrm{O} 7 \mathrm{~B}$ and $\mu_{2}-\mathrm{O} 8 B$, respectively, of the polyanion were found in a difference Fourier map and were freely refined. The $\mathrm{H}$ atoms of the $\mathrm{O} 6 \mathrm{~W}$ molecule were positioned geometrically and refined using a riding model (SHELXL2014 command HFIX 23), with $\mathrm{O}-\mathrm{H}=0.97 \AA$ and $U_{\text {iso }}(\mathrm{H})=1.5 U_{\text {eq }}(\mathrm{O})$. All other water $\mathrm{H}$ atoms were refined with distance restraints of $\mathrm{O}-\mathrm{H}=0.85$ (3) $\AA$ and $\mathrm{H} A \cdots \mathrm{H} B=1.35$ (3) $\AA$ using DFIX, and were included in the refinement with $U_{\text {iso }}(\mathrm{H})=1.5 U_{\text {eq }}(\mathrm{O})$. The unusually short $\mu_{2}-\mathrm{O} 17 \mathrm{~B} \cdots$ terminal-O $21 T^{\mathrm{i}}$ distance of 2.949 (5) $\AA$ (symmetry code as in Fig. 2.) is caused by the neighboring hydrogen bonds between the polyanions of the dimer as shown in Fig. 2. The highest peak in the difference map is $0.95 \AA$ from K4 and the largest hole is $0.92 \AA$ from Pt1.

\section{References}

Brandenburg, K. (1998). DIAMOND. Crystal Impact GbR, Bonn, Germany.

Brese, N. E. \& O'Keeffe, M. (1991). Acta Cryst. B47, 192-197.

Brown, I. D. \& Altermatt, D. (1985). Acta Cryst. B41, 244-247.

Farrugia, L. J. (2012). J. Appl. Cryst. 45, 849-854.

Joo, H.-C., Park, K.-M. \& Lee, U. (2011). Acta Cryst. E67, m1801$\mathrm{m} 1802$.

Konaka, S., Ozawa, Y., Shonaka, T., Watanabe, S. \& Yagasaki, A. (2011). Inorg. Chem. 50, 6183-6188.

Lee, U. (2006). Acta Cryst. E62, i176-i178.

Lee, U., Joo, H.-C., Park, K.-M., Mal, S. S., Kortz, U., Keita, B. \& Nadjo, L. (2008). Angew. Chem. Int. Ed. 47, 793-796.

Sheldrick, G. M. (2008). Acta Cryst. A64, 112-122.

Sheldrick, G. M. (2015). Acta Cryst. C71, 3-8.

Stoe \& Cie (1996). STADI4, X-RED and X-SHAPE. Stoe \& Cie Gmbh, Darmstadt, Germany. 


\section{supporting information}

Acta Cryst. (2015). E71, 647-649 [doi:10.1107/S2056989015009135]

\section{Crystal structure of pentapotassium dihydrogen nonavanadato(V)platinate(IV) nonahydrate}

\section{Hea-Chung Joo and Uk Lee}

\section{Computing details}

Data collection: STADI4 (Stoe \& Cie, 1996); cell refinement: STADI4 (Stoe \& Cie, 1996); data reduction: X-RED (Stoe \& Cie, 1996); program(s) used to solve structure: SHELXS2014 (Sheldrick, 2008); program(s) used to refine structure:

SHELXL2014 (Sheldrick, 2015); molecular graphics: ORTEP-3 for Windows (Farrugia, 2012) and DIAMOND

(Brandenburg, 1998); software used to prepare material for publication: SHELXL2014 (Sheldrick, 2015).

\section{Pentapotassium dihydrogen nonavanadato(V)platinate(IV) nonahydrate}

Crystal data

$\mathrm{K}_{5}\left[\mathrm{H}_{2} \mathrm{PtV}_{9} \mathrm{O}_{28}\right] \cdot 9 \mathrm{H}_{2} \mathrm{O}$

$M_{r}=1461.21$

Triclinic, $P \overline{1}$

$a=10.1663(7) \AA$

$b=12.8350(7) \AA$

$c=13.615(2) \AA$

$\alpha=103.734(5)^{\circ}$

$\beta=106.193(6)^{\circ}$

$\gamma=92.480(4)^{\circ}$

$V=1645.8(3) \AA^{3}$

\section{Data collection}

Stoe Stadi4 diffractometer

Radiation source: fine-focus sealed tube $\omega / 2-\theta$ scans

Absorption correction: empirical (using intensity measurements) (X-SHAPE; Stoe \& Cie, 1996)

$T_{\min }=0.301, T_{\max }=0.378$

6797 measured reflections

\section{Refinement}

Refinement on $F^{2}$

Least-squares matrix: full

$R\left[F^{2}>2 \sigma\left(F^{2}\right)\right]=0.028$

$w R\left(F^{2}\right)=0.066$

$S=1.10$

6797 reflections

526 parameters

25 restraints

$$
\begin{aligned}
& Z=2 \\
& F(000)=1392 \\
& D_{\mathrm{x}}=2.949 \mathrm{Mg} \mathrm{m}^{-3}
\end{aligned}
$$

Mo $K \alpha$ radiation, $\lambda=0.71069 \AA$

Cell parameters from 30 reflections

$\theta=9.6-10.5^{\circ}$

$\mu=7.42 \mathrm{~mm}^{-1}$

$T=298 \mathrm{~K}$

Block, dark brown

$0.21 \times 0.19 \times 0.17 \mathrm{~mm}$

6797 independent reflections 6242 reflections with $I>2 \sigma(I)$

$R_{\text {int }}=0.0000$

$\theta_{\max }=26.5^{\circ}, \theta_{\min }=1.6^{\circ}$

$h=-12 \rightarrow 12$

$k=-16 \rightarrow 15$

$l=0 \rightarrow 17$

3 standard reflections every $60 \mathrm{~min}$ intensity decay: $2.5 \%$

Primary atom site location: structure-invariant direct methods

Secondary atom site location: difference Fourier map

Hydrogen site location: difference Fourier map

$\mathrm{H}$ atoms treated by a mixture of independent and constrained refinement 
$w=1 /\left[\sigma^{2}\left(F_{\mathrm{o}}^{2}\right)+(0.0277 P)^{2}+4.5476 P\right]$

where $P=\left(F_{\mathrm{o}}^{2}+2 F_{\mathrm{c}}^{2}\right) / 3$

$(\Delta / \sigma)_{\max }<0.001$

$\Delta \rho_{\max }=1.30$ e $\AA^{-3}$
$\Delta \rho_{\min }=-1.46$ e $\AA^{-3}$

Extinction correction: SHELXL2014 (Sheldrick, 2015), $\mathrm{Fc}^{*}=\mathrm{kFc}\left[1+0.001 \times \mathrm{xF}^{2} \lambda^{3} / \sin (2 \theta)\right]^{-1 / 4}$

Extinction coefficient: 0.00505 (13)

\section{Special details}

Geometry. All esds (except the esd in the dihedral angle between two 1.s. planes) are estimated using the full covariance matrix. The cell esds are taken into account individually in the estimation of esds in distances, angles and torsion angles; correlations between esds in cell parameters are only used when they are defined by crystal symmetry. An approximate (isotropic) treatment of cell esds is used for estimating esds involving l.s. planes.

Fractional atomic coordinates and isotropic or equivalent isotropic displacement parameters $\left(\AA^{2}\right)$

\begin{tabular}{|c|c|c|c|c|}
\hline & $x$ & $y$ & $z$ & $U_{\text {iso }} * / U_{\text {eq }}$ \\
\hline Pt1 & $0.07476(2)$ & $0.66204(2)$ & $0.15945(2)$ & $0.01102(6)$ \\
\hline V1 & $0.08091(8)$ & $0.74803(6)$ & $-0.03864(6)$ & $0.01675(16)$ \\
\hline $\mathrm{V} 2$ & $0.05610(8)$ & $0.57644(6)$ & $0.35482(6)$ & $0.01798(16)$ \\
\hline V3 & $0.36393(8)$ & $0.67118(6)$ & $0.46862(6)$ & $0.01860(17)$ \\
\hline V4 & $0.38427(7)$ & $0.76066(6)$ & $0.27828(6)$ & $0.01375(15)$ \\
\hline V5 & $0.38558(8)$ & $0.84633(6)$ & $0.08300(6)$ & $0.01680(16)$ \\
\hline V6 & $0.15181(8)$ & 0.90837 (6) & $0.19135(6)$ & $0.01625(16)$ \\
\hline V7 & $0.14522(8)$ & $0.82358(6)$ & $0.38572(6)$ & $0.01838(17)$ \\
\hline V8 & $0.28571(8)$ & $0.50931(6)$ & $0.24210(6)$ & $0.01521(16)$ \\
\hline V9 & $0.30124(8)$ & $0.59746(6)$ & $0.04752(6)$ & $0.01441(15)$ \\
\hline K1 & $0.19325(16)$ & 0.94505 (11) & $0.81002(11)$ & $0.0433(3)$ \\
\hline K2 & $0.32640(13)$ & $0.27185(9)$ & $0.99666(11)$ & $0.0329(3)$ \\
\hline K3 & $0.29033(13)$ & $0.19821(10)$ & $0.25987(10)$ & 0.0353 \\
\hline K4 & $0.1688(2)$ & $0.15936(14)$ & $0.5300(2)$ & $0.0819(7)$ \\
\hline K5 & $0.33393(14)$ & $0.48579(12)$ & $0.61615(13)$ & $0.0439(3)$ \\
\hline O1D & $0.2234(3)$ & $0.7454(2)$ & $0.1318(2)$ & $0.0148(6)$ \\
\hline $\mathrm{O} 2 \mathrm{D}$ & 0.2133 & $0.6723(2)$ & $0.2981(2)$ & $0.0148(6)$ \\
\hline $\mathrm{O} 3 \mathrm{C}$ & $0.0321(3)$ & $0.8071(2)$ & $0.2320(2)$ & $0.0156(6)$ \\
\hline $\mathrm{O} 4 \mathrm{C}$ & $0.1590(3)$ & $0.5298(2)$ & $0.1039(2)$ & $0.0137(6)$ \\
\hline $\mathrm{O} 5 \mathrm{C}$ & 0.2793 & $0.8770(2)$ & $0.3209(2)$ & $0.0165(6)$ \\
\hline $\mathrm{O} 6 \mathrm{C}$ & $0.3986(3)$ & $0.6174(2)$ & $0.2014(2)$ & 0.0147 (6) \\
\hline O7B & -0.0627 & $0.5793(3)$ & 0.2053 & $0.0159(6)$ \\
\hline H7 & $-0.086(8)$ & $0.519(6)$ & $0.152(6)$ & $0.07(2)^{*}$ \\
\hline O8B & -0.0508 & $0.6659(3)$ & $0.0160(3)$ & $0.0168(7)$ \\
\hline H8 & $-0.067(6)$ & $0.609(5)$ & $-0.023(5)$ & $0.021(15)^{*}$ \\
\hline O9B & 0.2105 & $0.5994(3)$ & 0.4730 & $0.0202(7)$ \\
\hline O10B & $0.4757(3)$ & 0.7538 & $0.3997(3)$ & $0.0207(7)$ \\
\hline O11B & 0.4856 & $0.8317(3)$ & 0.2321 & $0.0179(7)$ \\
\hline O12B & 0.2409 & $0.8257(3)$ & $-0.0358(3)$ & $0.0197(7)$ \\
\hline O13B & 0.0439 & $0.8680(2)$ & $0.0548(3)$ & $0.0182(7)$ \\
\hline O14B & 0.3033 & $0.9526(2)$ & 0.1585 & $0.0174(6)$ \\
\hline O15B & $0.2871(3)$ & 0.8035 & $0.4919(3)$ & $0.0215(7)$ \\
\hline O16B & 0.0221 & $0.7196(3)$ & $0.3937(3)$ & $0.0204(7)$ \\
\hline O17B & $0.1402(3)$ & $0.4691(2)$ & 0.2815 & $0.0176(6)$ \\
\hline O18B & 0.4015 & $0.5523(3)$ & $0.3774(3)$ & $0.0184(7)$ \\
\hline
\end{tabular}




\begin{tabular}{|c|c|c|c|c|}
\hline O19B & $0.1590(3)$ & $0.6178(2)$ & -0.0635 (2) & $0.0158(6)$ \\
\hline $\mathrm{O} 20 \mathrm{~B}$ & $0.4224(3)$ & $0.7028(3)$ & $0.0424(3)$ & $0.0179(7)$ \\
\hline $\mathrm{O} 21 \mathrm{~T}$ & $-0.0224(4)$ & $0.7505(3)$ & $-0.1503(3)$ & $0.0270(8)$ \\
\hline $\mathrm{O} 22 \mathrm{~T}$ & $-0.0570(4)$ & $0.5099(3)$ & $0.3842(3)$ & $0.0257(8)$ \\
\hline $\mathrm{O} 23 \mathrm{~T}$ & $0.4743(4)$ & 0.6778 (3) & $0.5812(3)$ & $0.0287(8)$ \\
\hline $\mathrm{O} 24 \mathrm{~T}$ & $0.5067(3)$ & $0.9142(3)$ & $0.0618(3)$ & $0.0238(7)$ \\
\hline $\mathrm{O} 25 \mathrm{~T}$ & $0.0976(4)$ & $1.0199(3)$ & $0.2403(3)$ & $0.0245(7)$ \\
\hline $\mathrm{O} 26 \mathrm{~T}$ & $0.0934(4)$ & $0.9340(3)$ & $0.4384(3)$ & $0.0301(8)$ \\
\hline $\mathrm{O} 27 \mathrm{~T}$ & 0.3379 (4) & $0.3970(3)$ & $0.1941(3)$ & $0.0237(7)$ \\
\hline $\mathrm{O} 28 \mathrm{~T}$ & $0.3493(4)$ & $0.4863(3)$ & $-0.0055(3)$ & $0.0234(7)$ \\
\hline $\mathrm{O} 1 \mathrm{~W}$ & $0.1060(6)$ & $0.7552(4)$ & $0.6420(4)$ & $0.0514(12)$ \\
\hline $\mathrm{H} 1 \mathrm{~A}$ & $0.154(8)$ & $0.721(6)$ & $0.681(5)$ & $0.077 *$ \\
\hline H1B & $0.133(8)$ & $0.750(7)$ & $0.589(4)$ & $0.077 *$ \\
\hline $\mathrm{O} 2 \mathrm{~W}$ & $0.3763(6)$ & $0.9426(4)$ & $0.6891(4)$ & $0.0618(15)$ \\
\hline $\mathrm{H} 2 \mathrm{~A}$ & $0.347(9)$ & $0.908(6)$ & $0.623(3)$ & $0.093 *$ \\
\hline $\mathrm{H} 2 \mathrm{~B}$ & $0.428(8)$ & $0.997(5)$ & $0.688(6)$ & $0.093^{*}$ \\
\hline $\mathrm{O} 3 \mathrm{~W}$ & $0.2228(5)$ & $0.1440(4)$ & $0.7752(6)$ & $0.0660(17)$ \\
\hline $\mathrm{H} 3 \mathrm{~A}$ & $0.143(5)$ & $0.161(7)$ & $0.775(8)$ & $0.099 *$ \\
\hline $\mathrm{H} 3 \mathrm{~B}$ & $0.268(7)$ & $0.197(5)$ & $0.770(8)$ & $0.099^{*}$ \\
\hline $\mathrm{O} 4 \mathrm{~W}$ & $0.2138(4)$ & $0.0846(3)$ & $0.0168(4)$ & $0.0356(9)$ \\
\hline $\mathrm{H} 4 \mathrm{~A}$ & $0.132(4)$ & $0.097(5)$ & 0.007 (6) & $0.053 *$ \\
\hline $\mathrm{H} 4 \mathrm{~B}$ & $0.224(6)$ & $0.029(4)$ & $0.038(6)$ & $0.053^{*}$ \\
\hline $\mathrm{O} 5 \mathrm{~W}$ & $0.5107(5)$ & $0.1657(4)$ & $0.1656(4)$ & $0.0495(12)$ \\
\hline $\mathrm{H} 5 \mathrm{~A}$ & $0.550(8)$ & $0.130(5)$ & $0.210(4)$ & $0.074 *$ \\
\hline H5B & $0.481(8)$ & $0.120(5)$ & $0.106(3)$ & $0.074 *$ \\
\hline O6W & $0.3583(5)$ & 0.0913 (4) & 0.4279 (4) & $0.0552(13)$ \\
\hline H6A & 0.3463 & 0.0134 & 0.4006 & $0.083 *$ \\
\hline H6B & 0.4520 & 0.1148 & 0.4737 & $0.083^{*}$ \\
\hline O7W & $0.3045(5)$ & 0.3465 (4) & $0.7411(4)$ & $0.0471(12)$ \\
\hline H7A & $0.242(5)$ & $0.367(6)$ & $0.769(6)$ & $0.071^{*}$ \\
\hline H7B & $0.373(5)$ & $0.394(5)$ & $0.774(6)$ & $0.071^{*}$ \\
\hline O8W & $0.3022(6)$ & $0.6482(5)$ & 0.7759 (4) & $0.0634(15)$ \\
\hline H8A & $0.375(6)$ & $0.694(6)$ & 0.797 (6) & $0.095^{*}$ \\
\hline H8B & $0.287(9)$ & $0.645(7)$ & $0.834(4)$ & $0.095^{*}$ \\
\hline O9W & $0.1770(7)$ & $0.3233(4)$ & $0.4164(5)$ & $0.0629(15)$ \\
\hline H9A & $0.265(3)$ & $0.341(8)$ & $0.444(7)$ & $0.094 *$ \\
\hline H9B & $0.159(8)$ & $0.357(7)$ & $0.368(5)$ & $0.094 *$ \\
\hline
\end{tabular}

Atomic displacement parameters $\left(\AA^{2}\right)$

\begin{tabular}{lllllll}
\hline & $U^{11}$ & $U^{22}$ & $U^{33}$ & $U^{12}$ & $U^{13}$ & $U^{23}$ \\
\hline Pt1 & $0.00977(9)$ & $0.00941(9)$ & $0.01224(9)$ & $-0.00006(5)$ & $0.00238(6)$ & $0.00097(6)$ \\
V1 & $0.0165(4)$ & $0.0157(4)$ & $0.0161(4)$ & $0.0005(3)$ & $0.0017(3)$ & $0.0046(3)$ \\
V2 & $0.0159(4)$ & $0.0202(4)$ & $0.0177(4)$ & $-0.0023(3)$ & $0.0051(3)$ & $0.0053(3)$ \\
V3 & $0.0183(4)$ & $0.0199(4)$ & $0.0148(4)$ & $-0.0021(3)$ & $0.0010(3)$ & $0.0047(3)$ \\
V4 & $0.0120(3)$ & $0.0126(3)$ & $0.0144(4)$ & $-0.0016(3)$ & $0.0020(3)$ & $0.0019(3)$ \\
V5 & $0.0162(4)$ & $0.0151(4)$ & $0.0194(4)$ & $-0.0013(3)$ & $0.0058(3)$ & $0.0050(3)$ \\
V6 & $0.0182(4)$ & $0.0106(3)$ & $0.0187(4)$ & $0.0017(3)$ & $0.0048(3)$ & $0.0022(3)$
\end{tabular}


supporting information

\begin{tabular}{|c|c|c|c|c|c|c|}
\hline V7 & $0.0231(4)$ & 0.0151 (4) & $0.0160(4)$ & $0.0024(3)$ & $0.0076(3)$ & $0.0000(3)$ \\
\hline V8 & 0.0168 (4) & $0.0121(3)$ & 0.0162 (4) & $0.0025(3)$ & $0.0041(3)$ & $0.0036(3)$ \\
\hline V9 & 0.0158 (4) & $0.0120(3)$ & 0.0157 (4) & 0.0015 (3) & $0.0060(3)$ & $0.0026(3)$ \\
\hline K1 & $0.0556(9)$ & 0.0407 (7) & $0.0386(7)$ & $0.0112(6)$ & $0.0207(7)$ & $0.0109(6)$ \\
\hline K2 & $0.0380(7)$ & $0.0222(6)$ & $0.0465(7)$ & $0.0055(5)$ & $0.0257(6)$ & $0.0078(5)$ \\
\hline K3 & $0.0327(6)$ & $0.0355(7)$ & $0.0352(7)$ & $-0.0054(5)$ & $0.0075(5)$ & $0.0090(5)$ \\
\hline K4 & $0.1103(16)$ & $0.0426(9)$ & $0.1207(18)$ & $0.0171(10)$ & $0.0879(15)$ & $0.0091(10)$ \\
\hline K5 & $0.0328(7)$ & $0.0496(8)$ & $0.0667(10)$ & $0.0148(6)$ & $0.0246(7)$ & $0.0346(7)$ \\
\hline O1D & $0.0146(15)$ & $0.0120(14)$ & $0.0172(16)$ & $-0.0012(12)$ & $0.0047(12)$ & $0.0033(12)$ \\
\hline $\mathrm{O} 2 \mathrm{D}$ & $0.0153(15)$ & $0.0137(15)$ & $0.0126(15)$ & $-0.0033(12)$ & $0.0026(12)$ & $0.0010(12)$ \\
\hline $\mathrm{O} 3 \mathrm{C}$ & $0.0157(15)$ & $0.0128(15)$ & $0.0167(16)$ & $0.0019(12)$ & $0.0059(12)$ & $-0.0007(12)$ \\
\hline $\mathrm{O} 4 \mathrm{C}$ & $0.0136(14)$ & $0.0106(14)$ & $0.0153(15)$ & $0.0004(11)$ & $0.0036(12)$ & $0.0017(12)$ \\
\hline $\mathrm{O} 5 \mathrm{C}$ & $0.0169(15)$ & $0.0136(15)$ & $0.0161(15)$ & $0.0002(12)$ & $0.0028(12)$ & $0.0012(12)$ \\
\hline $\mathrm{O} 6 \mathrm{C}$ & $0.0128(14)$ & $0.0135(15)$ & $0.0153(15)$ & $-0.0002(12)$ & $0.0012(12)$ & $0.0030(12)$ \\
\hline O7B & $0.0159(15)$ & $0.0150(16)$ & $0.0159(16)$ & $0.0004(12)$ & $0.0051(13)$ & $0.0022(13)$ \\
\hline O8B & $0.0147(15)$ & $0.0162(17)$ & $0.0156(16)$ & $-0.0006(13)$ & $-0.0005(13)$ & $0.0031(14)$ \\
\hline O9B & $0.0221(17)$ & $0.0206(17)$ & $0.0169(16)$ & $-0.0022(13)$ & $0.0055(13)$ & $0.0040(13)$ \\
\hline O10B & $0.0194(16)$ & $0.0191(16)$ & $0.0205(17)$ & $-0.0027(13)$ & $0.0026(13)$ & $0.0044(13)$ \\
\hline O11B & $0.0142(15)$ & $0.0180(16)$ & $0.0204(17)$ & $-0.0025(12)$ & $0.0038(13)$ & $0.0049(13)$ \\
\hline O12B & $0.0243(17)$ & $0.0174(16)$ & $0.0179(16)$ & $-0.0010(13)$ & $0.0055(14)$ & $0.0069(13)$ \\
\hline O13B & $0.0162(15)$ & $0.0129(15)$ & $0.0236(17)$ & 0.0007 (12) & $0.0031(13)$ & $0.0050(13)$ \\
\hline O14B & $0.0187(16)$ & $0.0124(15)$ & $0.0210(17)$ & $-0.0007(12)$ & $0.0061(13)$ & $0.0041(13)$ \\
\hline O15B & $0.0273(18)$ & $0.0197(17)$ & $0.0141(16)$ & $-0.0007(14)$ & $0.0062(14)$ & $-0.0017(13)$ \\
\hline O16B & $0.0226(17)$ & $0.0192(16)$ & $0.0204(17)$ & $0.0025(13)$ & $0.0107(14)$ & $0.0018(13)$ \\
\hline O17B & $0.0190(16)$ & $0.0142(15)$ & $0.0185(16)$ & $-0.0015(12)$ & $0.0049(13)$ & $0.0039(13)$ \\
\hline O18B & $0.0169(15)$ & $0.0173(16)$ & $0.0185(16)$ & $0.0016(12)$ & 0.0009 (13) & $0.0052(13)$ \\
\hline O19B & $0.0167(15)$ & $0.0144(15)$ & $0.0138(15)$ & $0.0029(12)$ & $0.0022(12)$ & $0.0014(12)$ \\
\hline $\mathrm{O} 20 \mathrm{~B}$ & $0.0180(16)$ & $0.0178(16)$ & $0.0200(17)$ & $0.0031(13)$ & 0.0085 (13) & $0.0054(13)$ \\
\hline $\mathrm{O} 21 \mathrm{~T}$ & $0.0298(19)$ & $0.0255(18)$ & $0.0220(18)$ & $0.0016(15)$ & $0.0007(15)$ & $0.0075(15)$ \\
\hline $\mathrm{O} 22 \mathrm{~T}$ & $0.0233(18)$ & $0.0306(19)$ & $0.0266(19)$ & $-0.0029(15)$ & 0.0109 (15) & $0.0106(15)$ \\
\hline $\mathrm{O} 23 \mathrm{~T}$ & 0.0287 (19) & $0.033(2)$ & $0.0186(18)$ & $-0.0072(16)$ & $-0.0024(15)$ & $0.0079(15)$ \\
\hline $\mathrm{O} 24 \mathrm{~T}$ & $0.0224(17)$ & $0.0224(17)$ & $0.0281(19)$ & $-0.0033(14)$ & $0.0091(15)$ & $0.0086(15)$ \\
\hline $\mathrm{O} 25 \mathrm{~T}$ & $0.0245(18)$ & $0.0164(16)$ & $0.032(2)$ & $0.0048(14)$ & $0.0081(15)$ & 0.0041 (14) \\
\hline $\mathrm{O} 26 \mathrm{~T}$ & $0.039(2)$ & $0.0213(18)$ & $0.029(2)$ & $0.0061(16)$ & $0.0154(17)$ & $-0.0020(15)$ \\
\hline $\mathrm{O} 27 \mathrm{~T}$ & $0.0264(18)$ & $0.0180(17)$ & $0.0283(19)$ & $0.0074(14)$ & $0.0085(15)$ & 0.0077 (14) \\
\hline $\mathrm{O} 28 \mathrm{~T}$ & $0.0278(18)$ & $0.0164(16)$ & 0.0277 (19) & $0.0037(14)$ & $0.0130(15)$ & $0.0034(14)$ \\
\hline $\mathrm{O} 1 \mathrm{~W}$ & $0.066(3)$ & $0.050(3)$ & 0.041 (3) & 0.008 (2) & $0.025(3)$ & 0.007 (2) \\
\hline $\mathrm{O} 2 \mathrm{~W}$ & $0.080(4)$ & $0.056(3)$ & $0.035(3)$ & $-0.024(3)$ & $0.017(3)$ & $-0.010(2)$ \\
\hline $\mathrm{O} 3 \mathrm{~W}$ & $0.028(2)$ & $0.058(3)$ & $0.126(5)$ & $0.013(2)$ & $0.028(3)$ & $0.044(4)$ \\
\hline $\mathrm{O} 4 \mathrm{~W}$ & $0.028(2)$ & $0.035(2)$ & $0.054(3)$ & $0.0107(17)$ & $0.0133(19)$ & $0.027(2)$ \\
\hline $\mathrm{O} 5 \mathrm{~W}$ & $0.052(3)$ & $0.041(3)$ & 0.049 (3) & $-0.008(2)$ & $0.013(2)$ & $0.004(2)$ \\
\hline O6W & $0.051(3)$ & $0.034(2)$ & $0.056(3)$ & $-0.011(2)$ & $0.003(2)$ & $-0.014(2)$ \\
\hline O7W & $0.030(2)$ & $0.043(3)$ & $0.078(4)$ & 0.0107 (19) & $0.023(2)$ & $0.025(2)$ \\
\hline $\mathrm{O} 8 \mathrm{~W}$ & $0.080(4)$ & $0.066(4)$ & $0.042(3)$ & $-0.016(3)$ & $0.028(3)$ & $0.002(3)$ \\
\hline O9W & $0.095(4)$ & $0.051(3)$ & $0.056(3)$ & $0.025(3)$ & $0.029(3)$ & $0.028(3)$ \\
\hline
\end{tabular}


Geometric parameters $\left(\AA,{ }^{\circ}\right)$

\begin{tabular}{|c|c|c|c|}
\hline Pt1-V6 & $3.1213(8)$ & $\mathrm{V} 8-\mathrm{O} 27 \mathrm{~T}$ & $1.612(3)$ \\
\hline $\mathrm{Pt} 1-\mathrm{V} 8$ & $3.1262(8)$ & $\mathrm{V} 8-\mathrm{O} 17 \mathrm{~B}$ & $1.803(3)$ \\
\hline $\mathrm{Pt} 1-\mathrm{V} 9$ & $3.1359(8)$ & $\mathrm{V} 8-\mathrm{O} 18 \mathrm{~B}$ & $1.826(3)$ \\
\hline Pt1-V7 & $3.1480(9)$ & $\mathrm{V} 8-\mathrm{O} 6 \mathrm{C}$ & $2.033(3)$ \\
\hline Pt1-V4 & $3.1566(8)$ & $\mathrm{V} 8-\mathrm{O} 4 \mathrm{C}$ & $2.045(3)$ \\
\hline $\mathrm{Pt} 1-\mathrm{V} 2$ & $3.1574(9)$ & $\mathrm{V} 8-\mathrm{O} 2 \mathrm{D}$ & $2.278(3)$ \\
\hline $\mathrm{V} 1-\mathrm{V} 5$ & $3.1204(11)$ & $\mathrm{V} 9-\mathrm{O} 28 \mathrm{~T}$ & $1.601(3)$ \\
\hline $\mathrm{V} 1-\mathrm{V} 6$ & $3.1801(11)$ & $\mathrm{V} 9-\mathrm{O} 20 \mathrm{~B}$ & $1.816(3)$ \\
\hline V1-V9 & $3.1802(11)$ & $\mathrm{V} 9-\mathrm{O} 19 \mathrm{~B}$ & $1.856(3)$ \\
\hline $\mathrm{V} 2-\mathrm{V} 3$ & $3.1217(11)$ & $\mathrm{V} 9-\mathrm{O} 6 \mathrm{C}$ & $2.001(3)$ \\
\hline $\mathrm{V} 2-\mathrm{V} 7$ & $3.1566(11)$ & $\mathrm{V} 9-\mathrm{O} 4 \mathrm{C}$ & $2.064(3)$ \\
\hline $\mathrm{V} 2-\mathrm{V} 8$ & 3.1767 (11) & V9-O1D & $2.270(3)$ \\
\hline $\mathrm{V} 2-\mathrm{V} 4$ & $4.4982(11)$ & $\mathrm{K} 1-\mathrm{O} 3 \mathrm{~W}^{\mathrm{i}}$ & $2.725(5)$ \\
\hline $\mathrm{V} 3-\mathrm{V} 4$ & $3.1255(11)$ & $\mathrm{K} 1-\mathrm{O} 2 \mathrm{~W}$ & $2.804(6)$ \\
\hline $\mathrm{V} 3-\mathrm{V} 8$ & $3.1476(11)$ & $\mathrm{K} 1-\mathrm{O} 1 \mathrm{~W}$ & $2.827(5)$ \\
\hline $\mathrm{V} 3-\mathrm{V} 7$ & $3.1731(11)$ & $\mathrm{K} 1-\mathrm{O} 12 \mathrm{~B}^{\mathrm{ii}}$ & $2.830(3)$ \\
\hline V4-V5 & $3.1120(11)$ & $\mathrm{K} 1-\mathrm{O} 4 \mathrm{~W}^{\mathrm{iii}}$ & $2.903(5)$ \\
\hline V4-V9 & $3.1960(11)$ & $\mathrm{K} 1-\mathrm{O} 25 \mathrm{~T}^{\mathrm{iv}}$ & $2.924(4)$ \\
\hline V4-V7 & $3.2078(11)$ & $\mathrm{K} 1-\mathrm{O} 24 \mathrm{~T}^{\mathrm{v}}$ & $3.267(4)$ \\
\hline V4-V8 & $3.2162(10)$ & $\mathrm{K} 1-\mathrm{O} 5 \mathrm{~W}^{\mathrm{vi}}$ & $3.351(6)$ \\
\hline V5-V9 & $3.1578(10)$ & $\mathrm{K} 2-\mathrm{O} 4 \mathrm{~W}^{\mathrm{ii}}$ & $2.722(4)$ \\
\hline V5-V6 & $3.1654(11)$ & $\mathrm{K} 2-\mathrm{O} 27 \mathrm{~T}^{\mathrm{ii}}$ & $2.753(4)$ \\
\hline $\mathrm{V} 6-\mathrm{V} 7$ & $3.1068(12)$ & $\mathrm{K} 2-\mathrm{O} 28 \mathrm{~T}^{\mathrm{ii}}$ & $2.760(3)$ \\
\hline $\mathrm{V} 8-\mathrm{V} 9$ & $3.1566(11)$ & $\mathrm{K} 2-\mathrm{O} 20 \mathrm{~B}^{\mathrm{vi}}$ & $2.772(3)$ \\
\hline $\mathrm{Pt} 1-\mathrm{O} 1 \mathrm{D}$ & $1.985(3)$ & $\mathrm{K} 2-\mathrm{O} 8 \mathrm{~B}^{\mathrm{vii}}$ & $2.917(3)$ \\
\hline $\mathrm{Pt} 1-\mathrm{O} 2 \mathrm{D}$ & $1.986(3)$ & $\mathrm{K} 2-\mathrm{O} 3 \mathrm{~W}$ & $2.936(7)$ \\
\hline $\mathrm{Pt} 1-\mathrm{O} 4 \mathrm{C}$ & $2.015(3)$ & $\mathrm{K} 2-\mathrm{O} 24 \mathrm{~T}^{\mathrm{vi}}$ & 3.069 (4) \\
\hline $\mathrm{Pt} 1-\mathrm{O} 3 \mathrm{C}$ & $2.017(3)$ & $\mathrm{K} 2-\mathrm{O} 5 \mathrm{~W}^{\mathrm{ii}}$ & $3.156(5)$ \\
\hline $\mathrm{Pt} 1-\mathrm{O} 8 \mathrm{~B}$ & $2.027(3)$ & $\mathrm{K} 3-\mathrm{O} 23 \mathrm{~T}^{\mathrm{vi}}$ & $2.844(4)$ \\
\hline $\mathrm{Pt} 1-\mathrm{O} 7 \mathrm{~B}$ & $2.036(3)$ & $\mathrm{K} 3-\mathrm{O} 25 \mathrm{~T}^{\mathrm{viii}}$ & $2.860(4)$ \\
\hline $\mathrm{V} 1-\mathrm{O} 21 \mathrm{~T}$ & $1.598(3)$ & $\mathrm{K} 3-\mathrm{O} 5 \mathrm{~W}$ & $2.876(5)$ \\
\hline $\mathrm{V} 1-\mathrm{O} 12 \mathrm{~B}$ & $1.857(3)$ & $\mathrm{K} 3-\mathrm{O} 6 \mathrm{~W}$ & $2.878(6)$ \\
\hline $\mathrm{V} 1-\mathrm{O} 13 \mathrm{~B}$ & $1.876(3)$ & $\mathrm{K} 3-\mathrm{O} 9 \mathrm{~W}$ & $2.890(6)$ \\
\hline $\mathrm{V} 1-\mathrm{O} 19 \mathrm{~B}$ & $1.880(3)$ & $\mathrm{K} 3-\mathrm{O} 21 \mathrm{~T}^{\mathrm{ix}}$ & $2.904(4)$ \\
\hline $\mathrm{V} 1-\mathrm{O} 8 \mathrm{~B}$ & $2.064(3)$ & $\mathrm{K} 3-\mathrm{O} 27 \mathrm{~T}$ & $2.960(4)$ \\
\hline $\mathrm{V} 1-\mathrm{O} 1 \mathrm{D}$ & $2.377(3)$ & $\mathrm{K} 3-\mathrm{O} 4 \mathrm{~W}$ & $3.139(5)$ \\
\hline $\mathrm{V} 2-\mathrm{O} 22 \mathrm{~T}$ & $1.596(3)$ & $\mathrm{K} 3-\mathrm{O} 14 \mathrm{~B}^{\mathrm{viii}}$ & $3.151(3)$ \\
\hline $\mathrm{V} 2-\mathrm{O} 9 \mathrm{~B}$ & $1.862(3)$ & K4-O6W & $2.733(5)$ \\
\hline $\mathrm{V} 2-\mathrm{O} 16 \mathrm{~B}$ & $1.863(3)$ & $\mathrm{K} 4-\mathrm{O} 16 \mathrm{~B}^{\mathrm{vii}}$ & $2.806(4)$ \\
\hline $\mathrm{V} 2-\mathrm{O} 17 \mathrm{~B}$ & $1.885(3)$ & $\mathrm{K} 4-\mathrm{O} 26 \mathrm{~T}^{\mathrm{viii}}$ & $2.841(4)$ \\
\hline $\mathrm{V} 2-\mathrm{O} 7 \mathrm{~B}$ & $2.067(3)$ & K4-O9W & $2.904(5)$ \\
\hline $\mathrm{V} 2-\mathrm{O} 2 \mathrm{D}$ & $2.374(3)$ & $\mathrm{K} 4-\mathrm{O} 26 \mathrm{~T}^{\mathrm{vii}}$ & $3.055(4)$ \\
\hline $\mathrm{V} 3-\mathrm{O} 23 \mathrm{~T}$ & $1.608(3)$ & $\mathrm{K} 4-\mathrm{O} 7 \mathrm{~W}$ & $3.187(6)$ \\
\hline $\mathrm{V} 3-\mathrm{O} 9 \mathrm{~B}$ & $1.800(3)$ & $\mathrm{K} 4-\mathrm{O} 3 \mathrm{~W}$ & $3.284(7)$ \\
\hline $\mathrm{V} 3-\mathrm{O} 18 \mathrm{~B}$ & $1.853(3)$ & $\mathrm{K} 5-\mathrm{O} 8 \mathrm{~W}$ & $2.734(6)$ \\
\hline $\mathrm{V} 3-\mathrm{O} 15 \mathrm{~B}$ & $1.895(3)$ & $\mathrm{K} 5-\mathrm{O} 18 \mathrm{~B}^{\mathrm{vi}}$ & $2.736(3)$ \\
\hline
\end{tabular}


V3-O10B
V3-O2D
V4-O10B
V4-O11B
V4-O6C
V4-O5C
V4-O2D
V4-O1D
V5-O24T
V5-O12B
V5-O20B
V5-O14B
V5-O11B
V5-O1D
V6-O25T
V6-O13B
V6-O14B
V6-O3C
V6-O5C
V6-O1D
V7-O26T
V7-O15B
V7-O16B
V7-O5C
V7-O3C
V7-O2D

O1D-Pt1-O2D

$\mathrm{O} 1 \mathrm{D}-\mathrm{Pt} 1-\mathrm{O} 4 \mathrm{C}$

$\mathrm{O} 2 \mathrm{D}-\mathrm{Pt} 1-\mathrm{O} 4 \mathrm{C}$

O1D-Pt1-O3C

$\mathrm{O} 2 \mathrm{D}-\mathrm{Pt} 1-\mathrm{O} 3 \mathrm{C}$

$\mathrm{O} 4 \mathrm{C}-\mathrm{Pt} 1-\mathrm{O} 3 \mathrm{C}$

O1D-Pt1-O8B

$\mathrm{O} 2 \mathrm{D}-\mathrm{Pt} 1-\mathrm{O} 8 \mathrm{~B}$

$\mathrm{O} 4 \mathrm{C}-\mathrm{Pt} 1-\mathrm{O} 8 \mathrm{~B}$

$\mathrm{O} 3 \mathrm{C}-\mathrm{Pt} 1-\mathrm{O} 8 \mathrm{~B}$

O1D-Pt1-O7B

O2D-Pt1-O7B

$\mathrm{O} 4 \mathrm{C}-\mathrm{Pt} 1-\mathrm{O} 7 \mathrm{~B}$

$\mathrm{O} 3 \mathrm{C}-\mathrm{Pt} 1-\mathrm{O} 7 \mathrm{~B}$

O8B-Pt1-O7B

O1D-Pt1-V6

O2D-Pt1-V6

$\mathrm{O} 4 \mathrm{C}-\mathrm{Pt} 1-\mathrm{V} 6$

$\mathrm{O} 3 \mathrm{C}-\mathrm{Pt} 1-\mathrm{V} 6$

O8B-Pt1-V6

O7B-Pt1-V6
$2.064(3)$

$2.403(3)$

$1.681(3)$

$1.685(3)$

$1.921(3)$

$1.943(3)$

$2.148(3)$

$2.158(3)$

$1.607(3)$

$1.813(3)$

$1.878(3)$

$1.879(3)$

$2.058(3)$

$2.381(3)$

1.617 (3)

$1.812(3)$

1.824 (3)

2.014 (3)

2.015 (3)

$2.284(3)$

$1.610(3)$

$1.812(3)$

$1.832(3)$

1.997 (3)

2.043 (3)

2.269 (3)

$84.61(12)$

85.94 (12)

85.94 (12)

85.44 (12)

84.79 (12)

$167.89(12)$

88.49 (13)

172.89 (13)

95.27 (13)

92.99 (13)

$173.32(13)$

88.75 (12)

$94.36(12)$

$93.22(13)$

98.12 (13)

46.91 (9)

88.79 (9)

132.85 (9)

$39.21(9)$

$85.25(10)$

$132.36(9)$
$\mathrm{K} 5-\mathrm{O} 9 \mathrm{~B}$

$\mathrm{K} 5-\mathrm{O} 7 \mathrm{~W}$

$\mathrm{K} 5-\mathrm{O} 22 \mathrm{~T}^{\mathrm{vii}}$

$\mathrm{K} 5-\mathrm{O} 9 \mathrm{~W}$

$\mathrm{K} 5-\mathrm{O} 23 \mathrm{~T}$

$\mathrm{O} 7 \mathrm{~B}-\mathrm{H} 7$

O8B-H8

O1W-H1A

$\mathrm{O} 1 \mathrm{~W}-\mathrm{H} 1 \mathrm{~B}$

$\mathrm{O} 2 \mathrm{~W}-\mathrm{H} 2 \mathrm{~A}$

$\mathrm{O} 2 \mathrm{~W}-\mathrm{H} 2 \mathrm{~B}$

$\mathrm{O} 3 \mathrm{~W}-\mathrm{H} 3 \mathrm{~A}$

$\mathrm{O} 3 \mathrm{~W}-\mathrm{H} 3 \mathrm{~B}$

$\mathrm{O} 4 \mathrm{~W}-\mathrm{H} 4 \mathrm{~A}$

$\mathrm{O} 4 \mathrm{~W}-\mathrm{H} 4 \mathrm{~B}$

$\mathrm{O} 5 \mathrm{~W}-\mathrm{H} 5 \mathrm{~A}$

$\mathrm{O} 5 \mathrm{~W}-\mathrm{H} 5 \mathrm{~B}$

O6W-H6A

O6W-H6B

$\mathrm{O} 7 \mathrm{~W}-\mathrm{H} 7 \mathrm{~A}$

$\mathrm{O} 7 \mathrm{~W}-\mathrm{H} 7 \mathrm{~B}$

$\mathrm{O} 8 \mathrm{~W}-\mathrm{H} 8 \mathrm{~A}$

$\mathrm{O} 8 \mathrm{~W}-\mathrm{H} 8 \mathrm{~B}$

O9W-H9A

O9W-H9B

$\mathrm{Pt} 1-\mathrm{V} 4-\mathrm{V} 8$

$\mathrm{V} 9-\mathrm{V} 4-\mathrm{V} 8$

$\mathrm{V} 7-\mathrm{V} 4-\mathrm{V} 8$

$\mathrm{V} 4-\mathrm{V} 5-\mathrm{V} 1$

$\mathrm{V} 4-\mathrm{V} 5-\mathrm{V} 9$

$\mathrm{V} 1-\mathrm{V} 5-\mathrm{V} 9$

$\mathrm{V} 4-\mathrm{V} 5-\mathrm{V} 6$

$\mathrm{V} 1-\mathrm{V} 5-\mathrm{V} 6$

V9-V5-V6

V4-V5-Pt1

V1-V5-Pt1

V9-V5-Pt1

$\mathrm{V} 6-\mathrm{V} 5-\mathrm{Pt} 1$

$\mathrm{V} 7-\mathrm{V} 6-\mathrm{Pt} 1$

V7-V6-V5

Pt1-V6-V5

V7-V6-V1

$\mathrm{Pt} 1-\mathrm{V} 6-\mathrm{V} 1$

$\mathrm{V} 5-\mathrm{V} 6-\mathrm{V} 1$

$\mathrm{V} 6-\mathrm{V} 7-\mathrm{V} 3$

Pt1-V7-V3
2.764 (4)

$2.803(5)$

2.817 (4)

$2.982(7)$

2.996 (4)

$0.90(8)$

0.77 (6)

0.84 (3)

$0.83(3)$

$0.87(3)$

0.86 (3)

0.84 (3)

$0.83(3)$

0.83 (3)

0.83 (3)

0.87 (3)

$0.84(3)$

0.9700

0.9700

0.85 (3)

0.84 (3)

$0.86(3)$

$0.86(3)$

0.87 (3)

0.85 (3)

58.745 (19)

58.98 (2)

89.47 (3)

$92.76(3)$

61.29 (2)

60.86 (2)

62.06 (2)

60.78 (3)

$91.58(3)$

46.338 (17)

46.428 (18)

45.928 (17)

45.659 (17)

60.72 (2)

118.88 (3)

87.85 (2)

120.95 (3)

60.23 (2)

58.91 (2)

120.25 (3)

87.79 (2) 


\begin{tabular}{|c|c|c|c|}
\hline $\mathrm{O} 1 \mathrm{D}-\mathrm{Pt} 1-\mathrm{V} 8$ & $89.65(9)$ & $\mathrm{V} 2-\mathrm{V} 7-\mathrm{V} 3$ & $59.10(2)$ \\
\hline $\mathrm{O} 2 \mathrm{D}-\mathrm{Pt} 1-\mathrm{V} 8$ & $46.63(9)$ & $\mathrm{V} 6-\mathrm{V} 7-\mathrm{V} 4$ & $61.64(2)$ \\
\hline $\mathrm{O} 4 \mathrm{C}-\mathrm{Pt} 1-\mathrm{V} 8$ & $40.00(9)$ & $\mathrm{Pt} 1-\mathrm{V} 7-\mathrm{V} 4$ & $59.55(2)$ \\
\hline $\mathrm{O} 3 \mathrm{C}-\mathrm{Pt} 1-\mathrm{V} 8$ & $131.42(9)$ & $\mathrm{V} 2-\mathrm{V} 7-\mathrm{V} 4$ & $89.94(3)$ \\
\hline $\mathrm{O} 8 \mathrm{~B}-\mathrm{Pt} 1-\mathrm{V} 8$ & $135.22(10)$ & $\mathrm{V} 3-\mathrm{V} 7-\mathrm{V} 4$ & $58.66(2)$ \\
\hline $\mathrm{O} 7 \mathrm{~B}-\mathrm{Pt} 1-\mathrm{V} 8$ & $86.34(9)$ & $\mathrm{Pt} 1-\mathrm{V} 8-\mathrm{V} 3$ & $88.63(2)$ \\
\hline V6-Pt1-V8 & $123.63(2)$ & $\mathrm{Pt} 1-\mathrm{V} 8-\mathrm{V} 9$ & $59.88(2)$ \\
\hline $\mathrm{O} 1 \mathrm{D}-\mathrm{Pt} 1-\mathrm{V} 9$ & $46.16(9)$ & $\mathrm{V} 3-\mathrm{V} 8-\mathrm{V} 9$ & $119.00(3)$ \\
\hline $\mathrm{O} 2 \mathrm{D}-\mathrm{Pt} 1-\mathrm{V} 9$ & $89.07(9)$ & $\mathrm{V} 3-\mathrm{V} 8-\mathrm{V} 2$ & $59.15(2)$ \\
\hline $\mathrm{O} 4 \mathrm{C}-\mathrm{Pt} 1-\mathrm{V} 9$ & $40.35(9)$ & $\mathrm{V} 9-\mathrm{V} 8-\mathrm{V} 2$ & $120.00(3)$ \\
\hline $\mathrm{O} 3 \mathrm{C}-\mathrm{Pt} 1-\mathrm{V} 9$ & $131.59(9)$ & $\mathrm{V} 3-\mathrm{V} 8-\mathrm{V} 4$ & $58.82(2)$ \\
\hline $\mathrm{O} 8 \mathrm{~B}-\mathrm{Pt} 1-\mathrm{V} 9$ & $87.36(10)$ & $\mathrm{V} 9-\mathrm{V} 8-\mathrm{V} 4$ & $60.19(2)$ \\
\hline $\mathrm{O} 7 \mathrm{~B}-\mathrm{Pt} 1-\mathrm{V} 9$ & $134.69(9)$ & $\mathrm{V} 2-\mathrm{V} 8-\mathrm{V} 4$ & $89.43(3)$ \\
\hline $\mathrm{V} 6-\mathrm{Pt} 1-\mathrm{V} 9$ & $92.83(2)$ & $\mathrm{Pt} 1-\mathrm{V} 9-\mathrm{V} 8$ & $59.58(2)$ \\
\hline $\mathrm{V} 8-\mathrm{Pt} 1-\mathrm{V} 9$ & $60.54(2)$ & $\mathrm{Pt} 1-\mathrm{V} 9-\mathrm{V} 5$ & $87.73(2)$ \\
\hline $\mathrm{O} 1 \mathrm{D}-\mathrm{Pt1}-\mathrm{V} 7$ & $88.61(9)$ & $\mathrm{V} 8-\mathrm{V} 9-\mathrm{V} 5$ & $119.42(3)$ \\
\hline $\mathrm{O} 2 \mathrm{D}-\mathrm{Pt} 1-\mathrm{V} 7$ & $45.87(9)$ & $\mathrm{Pt} 1-\mathrm{V} 9-\mathrm{V} 1$ & $60.07(2)$ \\
\hline $\mathrm{O} 4 \mathrm{C}-\mathrm{Pt} 1-\mathrm{V} 7$ & $131.81(9)$ & $\mathrm{V} 8-\mathrm{V} 9-\mathrm{V} 1$ & $119.64(3)$ \\
\hline $\mathrm{O} 3 \mathrm{C}-\mathrm{Pt} 1-\mathrm{V} 7$ & $39.46(9)$ & $\mathrm{V} 5-\mathrm{V} 9-\mathrm{V} 1$ & $58.99(2)$ \\
\hline $\mathrm{O} 8 \mathrm{~B}-\mathrm{Pt} 1-\mathrm{V} 7$ & $132.43(10)$ & Pt1-V9-V4 & $59.80(2)$ \\
\hline O7B-Pt1-V7 & $86.22(9)$ & $\mathrm{V} 8-\mathrm{V} 9-\mathrm{V} 4$ & $60.83(2)$ \\
\hline V6-Pt1-V7 & $59.41(2)$ & $\mathrm{V} 5-\mathrm{V} 9-\mathrm{V} 4$ & $58.65(2)$ \\
\hline $\mathrm{V} 8-\mathrm{Pt} 1-\mathrm{V} 7$ & $92.22(2)$ & $\mathrm{V} 1-\mathrm{V} 9-\mathrm{V} 4$ & $90.08(3)$ \\
\hline V9-Pt1-V7 & $122.21(2)$ & $\mathrm{O} 3 \mathrm{~W}^{\mathrm{i}}-\mathrm{K} 1-\mathrm{O} 2 \mathrm{~W}$ & $71.45(18)$ \\
\hline $\mathrm{O} 1 \mathrm{D}-\mathrm{Pt} 1-\mathrm{V} 4$ & $42.45(9)$ & $\mathrm{O} 3 \mathrm{~W}^{\mathrm{i}}-\mathrm{K} 1-\mathrm{O} 1 \mathrm{~W}$ & $122.0(2)$ \\
\hline $\mathrm{O} 2 \mathrm{D}-\mathrm{Pt} 1-\mathrm{V} 4$ & $42.16(9)$ & $\mathrm{O} 2 \mathrm{~W}-\mathrm{K} 1-\mathrm{O} 1 \mathrm{~W}$ & $73.89(15)$ \\
\hline $\mathrm{O} 4 \mathrm{C}-\mathrm{Pt} 1-\mathrm{V} 4$ & $84.02(8)$ & $\mathrm{O} 3 \mathrm{~W}^{\mathrm{i}}-\mathrm{K} 1-\mathrm{O} 12 \mathrm{~B}^{\mathrm{ii}}$ & $145.45(18)$ \\
\hline $\mathrm{O} 3 \mathrm{C}-\mathrm{Pt} 1-\mathrm{V} 4$ & $83.88(9)$ & $\mathrm{O} 2 \mathrm{~W}-\mathrm{K} 1-\mathrm{O} 12 \mathrm{~B}^{\mathrm{ii}}$ & $118.85(16)$ \\
\hline $\mathrm{O} 8 \mathrm{~B}-\mathrm{Pt} 1-\mathrm{V} 4$ & $130.94(9)$ & $\mathrm{O} 1 \mathrm{~W}-\mathrm{K} 1-\mathrm{O} 12 \mathrm{~B}^{\mathrm{ii}}$ & $92.12(13)$ \\
\hline $\mathrm{O} 7 \mathrm{~B}-\mathrm{Pt} 1-\mathrm{V} 4$ & $130.91(9)$ & $\mathrm{O} 3 \mathrm{~W}^{\mathrm{i}}-\mathrm{K} 1-\mathrm{O} 4 \mathrm{~W}^{\mathrm{iii}}$ & $78.75(18)$ \\
\hline V6-Pt1-V4 & $62.06(2)$ & $\mathrm{O} 2 \mathrm{~W}-\mathrm{K} 1-\mathrm{O} 4 \mathrm{~W}^{\mathrm{iii}}$ & $128.72(14)$ \\
\hline $\mathrm{V} 8-\mathrm{Pt} 1-\mathrm{V} 4$ & $61.58(2)$ & $\mathrm{O} 1 \mathrm{~W}-\mathrm{K} 1-\mathrm{O} 4 \mathrm{~W}^{\mathrm{iii}}$ & $155.55(14)$ \\
\hline $\mathrm{V} 9-\mathrm{Pt} 1-\mathrm{V} 4$ & $61.05(2)$ & 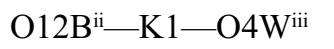 & $69.55(10)$ \\
\hline $\mathrm{V} 7-\mathrm{Pt} 1-\mathrm{V} 4$ & $61.17(2)$ & $\mathrm{O} 3 \mathrm{~W}^{\mathrm{i}}-\mathrm{K} 1-\mathrm{O} 25 \mathrm{~T}^{\mathrm{iv}}$ & $82.50(12)$ \\
\hline $\mathrm{O} 1 \mathrm{D}-\mathrm{Pt} 1-\mathrm{V} 2$ & $133.30(9)$ & $\mathrm{O} 2 \mathrm{~W}-\mathrm{K} 1-\mathrm{O} 25 \mathrm{~T}^{\mathrm{iv}}$ & $129.92(16)$ \\
\hline $\mathrm{O} 2 \mathrm{D}-\mathrm{Pt} 1-\mathrm{V} 2$ & $48.70(9)$ & $\mathrm{O} 1 \mathrm{~W}-\mathrm{K} 1-\mathrm{O} 25 \mathrm{~T}^{\mathrm{iv}}$ & $85.91(13)$ \\
\hline $\mathrm{O} 4 \mathrm{C}-\mathrm{Pt} 1-\mathrm{V} 2$ & $90.75(9)$ & $\mathrm{O} 12 \mathrm{~B}^{\mathrm{ii}}-\mathrm{K} 1-\mathrm{O} 25 \mathrm{~T}^{\mathrm{iv}}$ & $106.98(11)$ \\
\hline $\mathrm{O} 3 \mathrm{C}-\mathrm{Pt} 1-\mathrm{V} 2$ & $88.98(9)$ & $\mathrm{O} 4 \mathrm{~W}^{\mathrm{iii}}-\mathrm{K} 1-\mathrm{O} 25 \mathrm{~T}^{\mathrm{iv}}$ & $84.34(11)$ \\
\hline $\mathrm{O} 8 \mathrm{~B}-\mathrm{Pt} 1-\mathrm{V} 2$ & $138.14(9)$ & $\mathrm{O} 3 \mathrm{~W}^{\mathrm{i}}-\mathrm{K} 1-\mathrm{O} 24 \mathrm{~T}^{\mathrm{v}}$ & $65.13(14)$ \\
\hline $\mathrm{O} 7 \mathrm{~B}-\mathrm{Pt} 1-\mathrm{V} 2$ & $40.05(9)$ & $\mathrm{O} 2 \mathrm{~W}-\mathrm{K} 1-\mathrm{O} 24 \mathrm{~T}^{\mathrm{v}}$ & $65.37(12)$ \\
\hline $\mathrm{V} 6-\mathrm{Pt} 1-\mathrm{V} 2$ & $119.45(2)$ & $\mathrm{O} 1 \mathrm{~W}-\mathrm{K} 1-\mathrm{O} 24 \mathrm{~T}^{\mathrm{v}}$ & $133.36(13)$ \\
\hline $\mathrm{V} 8-\mathrm{Pt} 1-\mathrm{V} 2$ & $60.73(2)$ & $\mathrm{O} 12 \mathrm{~B}^{\mathrm{ii}}-\mathrm{K} 1-\mathrm{O} 24 \mathrm{~T}^{\mathrm{v}}$ & $88.44(10)$ \\
\hline $\mathrm{V} 9-\mathrm{Pt} 1-\mathrm{V} 2$ & $121.27(2)$ & $\mathrm{O} 4 \mathrm{~W}^{\mathrm{iii}}-\mathrm{K} 1-\mathrm{O} 24 \mathrm{~T}^{\mathrm{v}}$ & $64.45(10)$ \\
\hline $\mathrm{V} 7-\mathrm{Pt} 1-\mathrm{V} 2$ & $60.08(2)$ & $\mathrm{O} 25 \mathrm{~T}^{\mathrm{iv}}-\mathrm{K} 1-\mathrm{O} 24 \mathrm{~T}^{\mathrm{v}}$ & $138.04(10)$ \\
\hline $\mathrm{V} 4-\mathrm{Pt} 1-\mathrm{V} 2$ & $90.86(2)$ & $\mathrm{O} 3 \mathrm{~W}^{\mathrm{i}}-\mathrm{K} 1-\mathrm{O} 5 \mathrm{~W}^{\mathrm{vi}}$ & $110.45(14)$ \\
\hline $\mathrm{O} 21 \mathrm{~T}-\mathrm{V} 1-\mathrm{O} 12 \mathrm{~B}$ & $102.27(17)$ & $\mathrm{O} 2 \mathrm{~W}-\mathrm{K} 1-\mathrm{O} 5 \mathrm{~W}^{\mathrm{vi}}$ & $51.31(16)$ \\
\hline $\mathrm{O} 21 \mathrm{~T}-\mathrm{V} 1-\mathrm{O} 13 \mathrm{~B}$ & $102.73(17)$ & $\mathrm{O} 1 \mathrm{~W}-\mathrm{K} 1-\mathrm{O} 5 \mathrm{~W}^{\mathrm{vi}}$ & $79.54(14)$ \\
\hline $\mathrm{O} 12 \mathrm{~B}-\mathrm{V} 1-\mathrm{O} 13 \mathrm{~B}$ & $90.13(14)$ & 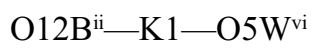 & $67.77(12)$ \\
\hline
\end{tabular}




$\begin{array}{ll}\text { O21T-V1-O19B } & 105.72(16) \\ \text { O12B-V1-O19B } & 91.05(14) \\ \text { O13B-V1-O19B } & 150.57(14) \\ \text { O21T-V1-O8B } & 99.44(16) \\ \text { O12B-V1-O8B } & 158.14(14) \\ \text { O13B-V1-O8B } & 82.64(14) \\ \text { O19B-V1-O8B } & 85.45(14) \\ \text { O21T-V1-O1D } & 176.60(16) \\ \text { O12B-V1-O1D } & 80.42(12) \\ \text { O13B-V1-O1D } & 75.06(12) \\ \text { O19B-V1-O1D } & 76.16(12) \\ \text { O8B-V1-O1D } & 77.79(12) \\ \text { O22T-V2-O9B } & 103.62(17) \\ \text { O22T-V2-O16B } & 104.45(17) \\ \text { O9B-V2-O16B } & 91.43(15) \\ \text { O22T-V2-O17B } & 104.04(17) \\ \text { O9B-V2-O17B } & 88.97(14) \\ \text { O16B-V2-O17B } & 150.56(14) \\ \text { O22T-V2-O7B } & 97.56(16) \\ \text { O9B-V2-O7B } & 158.78(14) \\ \text { O16B-V2-O7B } & 84.50(14) \\ \text { O17B-V2-O7B } & 84.59(13) \\ \text { O22T-V2-O2D } & 175.77(15) \\ \text { O9B-V2-O2D } & 80.53(13) \\ \text { O16B-V2-O2D } & 76.07(13) \\ \text { O17B-V2-O2D } & 74.97(12) \\ \text { O7B-V2-O2D } & 78.27(11) \\ \text { O23T-V3-O9B } & 104.10(17) \\ \text { O23T-V3-O18B } & 104.12(17) \\ \text { O9B-V3-O18B } & 92.55(15) \\ \text { O23T-V3-O15B } & 103.56(18) \\ \text { O9B-V3-O15B } & 90.80(15) \\ \text { O18B-V3-O15B } & 150.37(14) \\ \text { O23T-V3-O10B } & 101.92(16) \\ \text { O9B-V3-O10B } & 153.94(14) \\ \text { O18B-V3-O10B } & 82.41(14) \\ \text { O15B-V3-O10B } & 81.76(14) \\ \text { O23T-V3-O2D } & 174.90(16) \\ \text { O9B-V3-O2D } & 80.92(13) \\ \text { O18B-V3-O2D } & 76.28(12) \\ \text { O15B-V3-O2D } & 75.21(12) \\ \text { O10B-V3-O2D } & 73.04(12) \\ \text { O10B-V4-O11B } & 108.05(16) \\ \text { O10B-V4-O6C } & 98.10(15) \\ \text { O11B-V4-O6C } & 99.13(15) \\ \text { O10B-V4-O5C } & 98.12(15) \\ \text { O11B-V4-O5C } & 152.55(13) \\ \text { O6C-V4-O5C } & \\ \text { O1B } & \\ & \end{array}$

\begin{tabular}{|c|c|}
\hline $\mathrm{O} 4 \mathrm{~W}^{\mathrm{iii}}-\mathrm{K} 1-\mathrm{O} 5 \mathrm{~W}^{\mathrm{vi}}$ & $106.58(12)$ \\
\hline $\mathrm{O} 25 \mathrm{~T}^{\mathrm{iv}}-\mathrm{K} 1-\mathrm{O} 5 \mathrm{~W}^{\mathrm{vi}}$ & $164.20(12)$ \\
\hline $\mathrm{O} 24 \mathrm{~T}^{\mathrm{v}}-\mathrm{K} 1-\mathrm{O} 5 \mathrm{~W}^{\mathrm{vi}}$ & $57.73(10)$ \\
\hline $\mathrm{O} 4 \mathrm{~W}^{\mathrm{ii}}-\mathrm{K} 2-\mathrm{O} 27 \mathrm{~T}^{\mathrm{ii}}$ & $96.79(13)$ \\
\hline $\mathrm{O} 4 \mathrm{~W}^{\mathrm{ii}}-\mathrm{K} 2-\mathrm{O} 28 \mathrm{~T}^{\mathrm{ii}}$ & $157.70(12)$ \\
\hline $\mathrm{O} 27 \mathrm{~T}^{\mathrm{ii}}-\mathrm{K} 2-\mathrm{O} 28 \mathrm{~T}^{\mathrm{ii}}$ & $71.43(10)$ \\
\hline $\mathrm{O} 4 \mathrm{~W}^{\mathrm{ii}}-\mathrm{K} 2-\mathrm{O} 20 \mathrm{~B}^{\mathrm{vi}}$ & $124.36(11)$ \\
\hline $\mathrm{O} 27 \mathrm{~T}^{\mathrm{ii}}-\mathrm{K} 2-\mathrm{O} 20 \mathrm{~B}^{\mathrm{vi}}$ & $110.65(11)$ \\
\hline $\mathrm{O} 28 \mathrm{~T}^{\mathrm{ii}}-\mathrm{K} 2-\mathrm{O} 20 \mathrm{~B}^{\mathrm{vi}}$ & $77.91(10)$ \\
\hline $\mathrm{O} 4 \mathrm{~W}^{\mathrm{ii}}-\mathrm{K} 2-\mathrm{O} 8 \mathrm{~B}^{\mathrm{vii}}$ & $82.79(11)$ \\
\hline $\mathrm{O} 27 \mathrm{~T}^{\mathrm{ii}}-\mathrm{K} 2-\mathrm{O} 8 \mathrm{~B}^{\mathrm{vii}}$ & $73.02(10)$ \\
\hline $\mathrm{O} 28 \mathrm{~T}^{\mathrm{ii}}-\mathrm{K} 2-\mathrm{O} 8 \mathrm{~B}^{\mathrm{vii}}$ & $75.73(10)$ \\
\hline $\mathrm{O} 20 \mathrm{~B}^{\mathrm{vi}}-\mathrm{K} 2-\mathrm{O} 8 \mathrm{~B}^{\mathrm{vii}}$ & $150.46(10)$ \\
\hline $\mathrm{O} 4 \mathrm{~W}^{\mathrm{ii}}-\mathrm{K} 2-\mathrm{O} 3 \mathrm{~W}$ & $78.19(15)$ \\
\hline $\mathrm{O} 27 \mathrm{~T}^{\mathrm{ii}-\mathrm{K} 2-\mathrm{O} 3 \mathrm{~W}}$ & $162.23(13)$ \\
\hline $\mathrm{O} 28 \mathrm{~T}^{\mathrm{ii}}-\mathrm{K} 2-\mathrm{O} 3 \mathrm{~W}$ & $107.12(13)$ \\
\hline $\mathrm{O} 20 \mathrm{~B}^{\mathrm{vi}}-\mathrm{K} 2-\mathrm{O} 3 \mathrm{~W}$ & $85.81(12)$ \\
\hline $\mathrm{O} 8 \mathrm{~B}^{\mathrm{vii}}-\mathrm{K} 2-\mathrm{O} 3 \mathrm{~W}$ & $89.37(12)$ \\
\hline $\mathrm{O} 4 \mathrm{~W}^{\mathrm{ii}}-\mathrm{K} 2-\mathrm{O} 24 \mathrm{~T}^{\mathrm{vi}}$ & $69.29(11)$ \\
\hline $\mathrm{O} 27 \mathrm{~T}^{\mathrm{ii}}-\mathrm{K} 2-\mathrm{O} 24 \mathrm{~T}^{\mathrm{vi}}$ & $128.86(11)$ \\
\hline $\mathrm{O} 28 \mathrm{~T}^{\mathrm{ii}}-\mathrm{K} 2-\mathrm{O} 24 \mathrm{~T}^{\mathrm{vi}}$ & $132.88(10)$ \\
\hline $\mathrm{O} 20 \mathrm{~B}^{\mathrm{vi}}-\mathrm{K} 2-\mathrm{O} 24 \mathrm{~T}^{\mathrm{vi}}$ & $55.63(9)$ \\
\hline $\mathrm{O} 8 \mathrm{~B}^{\mathrm{vii}}-\mathrm{K} 2-\mathrm{O} 24 \mathrm{~T}^{\mathrm{vi}}$ & $145.32(10)$ \\
\hline $\mathrm{O} 3 \mathrm{~W}-\mathrm{K} 2-\mathrm{O} 24 \mathrm{~T}^{\mathrm{vi}}$ & $65.65(11)$ \\
\hline $\mathrm{O} 4 \mathrm{~W}^{\mathrm{ii}}-\mathrm{K} 2-\mathrm{O} 5 \mathrm{~W}^{\mathrm{ii}}$ & $63.16(12)$ \\
\hline $\mathrm{O} 27 \mathrm{~T}^{\mathrm{ii}}-\mathrm{K} 2-\mathrm{O} 5 \mathrm{~W}^{\mathrm{ii}}$ & $72.57(12)$ \\
\hline $\mathrm{O} 28 \mathrm{~T}^{\mathrm{ii}}-\mathrm{K} 2-\mathrm{O} 5 \mathrm{~W}^{\mathrm{ii}}$ & $126.96(12)$ \\
\hline $\mathrm{O} 20 \mathrm{~B}^{\mathrm{vi}}-\mathrm{K} 2-\mathrm{O} 5 \mathrm{~W}^{\mathrm{ii}}$ & $79.90(12)$ \\
\hline $\mathrm{O} 8 \mathrm{~B}^{\mathrm{vii}}-\mathrm{K} 2-\mathrm{O} 5 \mathrm{~W}^{\mathrm{ii}}$ & $127.23(12)$ \\
\hline $\mathrm{O} 3 \mathrm{~W}-\mathrm{K} 2-\mathrm{O} 5 \mathrm{~W}^{\mathrm{ii}}$ & $118.55(13)$ \\
\hline $\mathrm{O} 24 \mathrm{~T}^{\mathrm{vi}}-\mathrm{K} 2-\mathrm{O} 5 \mathrm{~W}^{\mathrm{ii}}$ & $57.03(11)$ \\
\hline $\mathrm{O} 23 \mathrm{~T}^{\mathrm{vi}}-\mathrm{K} 3-\mathrm{O} 25 \mathrm{~T}^{\mathrm{viii}}$ & $136.47(11)$ \\
\hline $\mathrm{O} 23 \mathrm{~T}^{\mathrm{vi}}-\mathrm{K} 3-\mathrm{O} 5 \mathrm{~W}$ & $75.77(13)$ \\
\hline $\mathrm{O} 25 \mathrm{~T}^{\mathrm{viii}}-\mathrm{K} 3-\mathrm{O} 5 \mathrm{~W}$ & $120.42(12)$ \\
\hline $\mathrm{O} 23 \mathrm{~T}^{\mathrm{vi}}-\mathrm{K} 3-\mathrm{O} 6 \mathrm{~W}$ & $72.84(12)$ \\
\hline $\mathrm{O} 25 \mathrm{~T}^{\mathrm{viii}}-\mathrm{K} 3-\mathrm{O} 6 \mathrm{~W}$ & $64.17(12)$ \\
\hline $\mathrm{O} 5 \mathrm{~W}-\mathrm{K} 3-\mathrm{O} 6 \mathrm{~W}$ & $104.30(15)$ \\
\hline $\mathrm{O} 23 \mathrm{~T}^{\mathrm{vi}}-\mathrm{K} 3-\mathrm{O} 9 \mathrm{~W}$ & $76.16(15)$ \\
\hline $\mathrm{O} 25 \mathrm{~T}^{\mathrm{viii}}-\mathrm{K} 3-\mathrm{O} 9 \mathrm{~W}$ & $87.40(14)$ \\
\hline $\mathrm{O} 5 \mathrm{~W}-\mathrm{K} 3-\mathrm{O} 9 \mathrm{~W}$ & $150.12(16)$ \\
\hline $\mathrm{O} 6 \mathrm{~W}-\mathrm{K} 3-\mathrm{O} 9 \mathrm{~W}$ & $76.75(14)$ \\
\hline $\mathrm{O} 23 \mathrm{~T}^{\mathrm{vi}}-\mathrm{K} 3-\mathrm{O} 21 \mathrm{~T}^{\mathrm{ix}}$ & $133.79(11)$ \\
\hline $\mathrm{O} 25 \mathrm{~T}^{\mathrm{viii}}-\mathrm{K} 3-\mathrm{O} 21 \mathrm{~T}^{\mathrm{ix}}$ & $74.44(10)$ \\
\hline $\mathrm{O} 5 \mathrm{~W}-\mathrm{K} 3-\mathrm{O} 21 \mathrm{~T}^{\mathrm{ix}}$ & $123.14(14)$ \\
\hline $\mathrm{O} 6 \mathrm{~W}-\mathrm{K} 3-\mathrm{O} 21 \mathrm{~T}^{\mathrm{ix}}$ & $128.44(13)$ \\
\hline $\mathrm{O} 9 \mathrm{~W}-\mathrm{K} 3-\mathrm{O} 21 \mathrm{~T}^{\mathrm{ix}}$ & $71.85(14)$ \\
\hline $\mathrm{O} 23 \mathrm{~T}^{\mathrm{vi}}-\mathrm{K} 3-\mathrm{O} 27 \mathrm{~T}$ & $74.05(10)$ \\
\hline $\mathrm{O} 25 \mathrm{~T}^{\mathrm{viii}}-\mathrm{K} 3-\mathrm{O} 27 \mathrm{~T}$ & $146.54(11)$ \\
\hline
\end{tabular}




$\begin{array}{ll}\text { O10B-V4-O2D } & 87.63(14) \\ \text { O11B-V4-O2D } & 164.23(14) \\ \text { O6C-V4-O2D } & 79.74(12) \\ \text { O5C-V4-O2D } & 78.95(12) \\ \text { O10B-V4-O1D } & 164.37(14) \\ \text { O11B-V4-O1D } & 87.58(14) \\ \text { O6C-V4-O1D } & 79.38(12) \\ \text { O5C-V4-O1D } & 79.03(12) \\ \text { O2D-V4-O1D } & 76.74(12) \\ \text { O24T-V5-O12B } & 104.93(17) \\ \text { O24T-V5-O20B } & 103.26(16) \\ \text { O12B-V5-O20B } & 91.36(15) \\ \text { O24T-V5-O14B } & 103.76(16) \\ \text { O12B-V5-O14B } & 91.43(15) \\ \text { O20B-V5-O14B } & 151.11(14) \\ \text { O24T-V5-O11B } & 100.00(16) \\ \text { O12B-V5-O11B } & 155.07(14) \\ \text { O20B-V5-O11B } & 82.61(14) \\ \text { O14B-V5-O11B } & 82.82(14) \\ \text { O24T-V5-O1D } & 173.91(15) \\ \text { O12B-V5-O1D } & 81.14(13) \\ \text { O20B-V5-O1D } & 75.91(12) \\ \text { O14B-V5-O1D } & 76.13(12) \\ \text { O11B-V5-O1D } & 73.92(11) \\ \text { O25T-V6-O13B } & 103.58(16) \\ \text { O25T-V6-O14B } & 103.90(16) \\ \text { O13B-V6-O14B } & 94.04(15) \\ \text { O25T-V6-O3C } & 97.90(16) \\ \text { O13B-V6-O3C } & 91.22(14) \\ \text { O14B-V6-O3C } & 155.66(14) \\ \text { O25T-V6-O5C } & 102.60(16) \\ \text { O13B-V6-O5C } & 152.25(14) \\ \text { O14B-V6-O5C } & 88.47(14) \\ \text { O3C-V6-O5C } & 76.20(13) \\ \text { O25T-V6-O1D } & 175.51(15) \\ \text { O13B-V6-O1D } & 78.63(13) \\ \text { O14B-V6-O1D } & 79.71(12) \\ \text { O3C-V6-O1D } & 78.07(12) \\ \text { O5C-V6-O1D } & 74.63(12) \\ \text { O26T-V7-O15B } & 103.92(18) \\ \text { O26T-V7-O16B } & 102.74(18) \\ \text { O15B-V7-O16B } & 95.34(16) \\ \text { O26T-V7-O5C } & 102.03(17) \\ \text { O15B-V7-O5C } & 89.99(14) \\ \text { O16B-V7-O5C } & 152.57(14) \\ \text { O26T-V7-O3C } & 98.14(17) \\ \text { O15B-V7-O3C } & \\ \text { O16B-V7-O3C } & \\ \text { O14) } & \\ & \end{array}$

\begin{tabular}{|c|c|}
\hline $\mathrm{O} 5 \mathrm{~W}-\mathrm{K} 3-\mathrm{O} 27 \mathrm{~T}$ & $73.96(12)$ \\
\hline $\mathrm{O} 6 \mathrm{~W}-\mathrm{K} 3-\mathrm{O} 27 \mathrm{~T}$ & $146.13(12)$ \\
\hline $\mathrm{O} 9 \mathrm{~W}-\mathrm{K} 3-\mathrm{O} 27 \mathrm{~T}$ & $88.67(13)$ \\
\hline 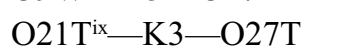 & $72.77(10)$ \\
\hline 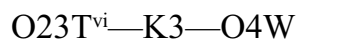 & $136.36(11)$ \\
\hline 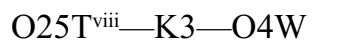 & $78.53(11)$ \\
\hline $\mathrm{O} 5 \mathrm{~W}-\mathrm{K} 3-\mathrm{O} 4 \mathrm{~W}$ & $61.86(12)$ \\
\hline $\mathrm{O} 6 \mathrm{~W}-\mathrm{K} 3-\mathrm{O} 4 \mathrm{~W}$ & $125.62(12)$ \\
\hline $\mathrm{O} 9 \mathrm{~W}-\mathrm{K} 3-\mathrm{O} 4 \mathrm{~W}$ & $141.67(15)$ \\
\hline $\mathrm{O} 21 \mathrm{~T}^{\mathrm{ix}}-\mathrm{K} 3-\mathrm{O} 4 \mathrm{~W}$ & $70.07(10)$ \\
\hline $\mathrm{O} 27 \mathrm{~T}-\mathrm{K} 3-\mathrm{O} 4 \mathrm{~W}$ & $84.26(10)$ \\
\hline 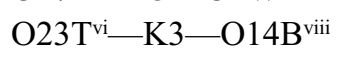 & $119.30(11)$ \\
\hline $\mathrm{O} 25 \mathrm{~T}^{\mathrm{viii}}-\mathrm{K} 3-\mathrm{O} 14 \mathrm{~B}^{\mathrm{viii}}$ & $53.38(9)$ \\
\hline $\mathrm{O} 5 \mathrm{~W}-\mathrm{K} 3-\mathrm{O} 14 \mathrm{~B}^{\mathrm{viii}}$ & $67.26(11)$ \\
\hline $\mathrm{O} 6 \mathrm{~W}-\mathrm{K} 3-\mathrm{O} 14 \mathrm{~B}^{\text {viii }}$ & $72.00(11)$ \\
\hline $\mathrm{O} 9 \mathrm{~W}-\mathrm{K} 3-\mathrm{O} 14 \mathrm{~B}^{\mathrm{viii}}$ & $137.58(13)$ \\
\hline $\mathrm{O} 21 \mathrm{~T}^{\mathrm{ix}}-\mathrm{K} 3-\mathrm{O} 14 \mathrm{~B}^{\mathrm{viii}}$ & $106.80(10)$ \\
\hline $\mathrm{O} 27 \mathrm{~T}-\mathrm{K} 3-\mathrm{O} 14 \mathrm{~B}^{\mathrm{viii}}$ & $132.52(10)$ \\
\hline $\mathrm{O} 4 \mathrm{~W}-\mathrm{K} 3-\mathrm{O} 14 \mathrm{~B}^{\mathrm{viii}}$ & $53.88(9)$ \\
\hline $\mathrm{O} 6 \mathrm{~W}-\mathrm{K} 4-\mathrm{O} 16 \mathrm{~B}^{\mathrm{vii}}$ & $162.73(15)$ \\
\hline $\mathrm{O} 6 \mathrm{~W}-\mathrm{K} 4-\mathrm{O} 26 \mathrm{~T}^{\text {viii }}$ & $73.88(13)$ \\
\hline $\mathrm{O} 16 \mathrm{~B}^{\mathrm{vii}}-\mathrm{K} 4-\mathrm{O} 26 \mathrm{~T}^{\mathrm{viii}}$ & $118.24(13)$ \\
\hline $\mathrm{O} 6 \mathrm{~W}-\mathrm{K} 4-\mathrm{O} 9 \mathrm{~W}$ & $78.81(17)$ \\
\hline $\mathrm{O} 16 \mathrm{~B}^{\mathrm{vii}}-\mathrm{K} 4-\mathrm{O} 9 \mathrm{~W}$ & $83.95(15)$ \\
\hline 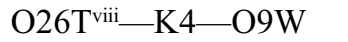 & $126.27(17)$ \\
\hline $\mathrm{O} 6 \mathrm{~W}-\mathrm{K} 4-\mathrm{O} 26 \mathrm{~T}^{\mathrm{vii}}$ & $138.56(13)$ \\
\hline $\mathrm{O} 16 \mathrm{~B}^{\mathrm{vii}}-\mathrm{K} 4-\mathrm{O} 26 \mathrm{~T}^{\mathrm{vii}}$ & $54.50(10)$ \\
\hline $\mathrm{O} 26 \mathrm{~T}^{\mathrm{viii}}-\mathrm{K} 4-\mathrm{O} 26 \mathrm{~T}^{\mathrm{vii}}$ & $64.75(12)$ \\
\hline $\mathrm{O} 9 \mathrm{~W}-\mathrm{K} 4-\mathrm{O} 26 \mathrm{~T}^{\mathrm{vii}}$ & $124.14(16)$ \\
\hline $\mathrm{O} 6 \mathrm{~W}-\mathrm{K} 4-\mathrm{O} 7 \mathrm{~W}$ & $111.55(14)$ \\
\hline $\mathrm{O} 16 \mathrm{~B}^{\mathrm{vii}}-\mathrm{K} 4-\mathrm{O} 7 \mathrm{~W}$ & $65.92(11)$ \\
\hline 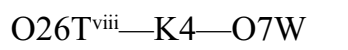 & $146.61(15)$ \\
\hline $\mathrm{O} 9 \mathrm{~W}-\mathrm{K} 4-\mathrm{O} 7 \mathrm{~W}$ & $86.49(16)$ \\
\hline 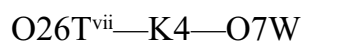 & $104.46(12)$ \\
\hline $\mathrm{O} 6 \mathrm{~W}-\mathrm{K} 4-\mathrm{O} 3 \mathrm{~W}$ & $118.81(16)$ \\
\hline $\mathrm{O} 16 \mathrm{~B}^{\mathrm{vii}}-\mathrm{K} 4-\mathrm{O} 3 \mathrm{~W}$ & $74.21(12)$ \\
\hline $\mathrm{O} 26 \mathrm{~T}^{\mathrm{viii}-\mathrm{K} 4-\mathrm{O} 3 \mathrm{~W}}$ & $95.01(14)$ \\
\hline $\mathrm{O} 9 \mathrm{~W}-\mathrm{K} 4-\mathrm{O} 3 \mathrm{~W}$ & $138.71(17)$ \\
\hline $\mathrm{O} 26 \mathrm{~T}^{\mathrm{vii}-\mathrm{K} 4-\mathrm{O} 3 \mathrm{~W}}$ & $68.97(12)$ \\
\hline $\mathrm{O} 7 \mathrm{~W}-\mathrm{K} 4-\mathrm{O} 3 \mathrm{~W}$ & $52.73(14)$ \\
\hline $\mathrm{O} 8 \mathrm{~W}-\mathrm{K} 5-\mathrm{O} 18 \mathrm{~B}^{\mathrm{vi}}$ & $116.68(16)$ \\
\hline $\mathrm{O} 8 \mathrm{~W}-\mathrm{K} 5-\mathrm{O} 9 \mathrm{~B}$ & $88.96(16)$ \\
\hline $\mathrm{O} 18 \mathrm{~B}^{\mathrm{vi}}-\mathrm{K} 5-\mathrm{O} 9 \mathrm{~B}$ & $115.20(11)$ \\
\hline $\mathrm{O} 8 \mathrm{~W}-\mathrm{K} 5-\mathrm{O} 7 \mathrm{~W}$ & $86.37(17)$ \\
\hline $\mathrm{O} 18 \mathrm{~B}^{\mathrm{vi}-\mathrm{K} 5-\mathrm{O} 7 \mathrm{~W}}$ & $94.58(12)$ \\
\hline $\mathrm{O} 9 \mathrm{~B}-\mathrm{K} 5-\mathrm{O} 7 \mathrm{~W}$ & $148.49(12)$ \\
\hline $\mathrm{O} 8 \mathrm{~W}-\mathrm{K} 5-\mathrm{O} 22 \mathrm{~T}^{\mathrm{vii}}$ & $67.49(15)$ \\
\hline $\mathrm{O} 18 \mathrm{~B}^{\mathrm{vi}}-\mathrm{K} 5-\mathrm{O} 22 \mathrm{~T}^{\mathrm{vii}}$ & $170.27(11)$ \\
\hline
\end{tabular}




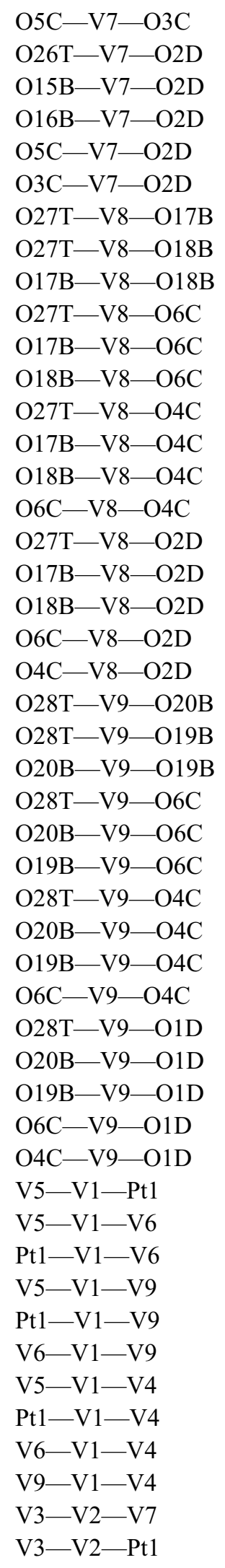

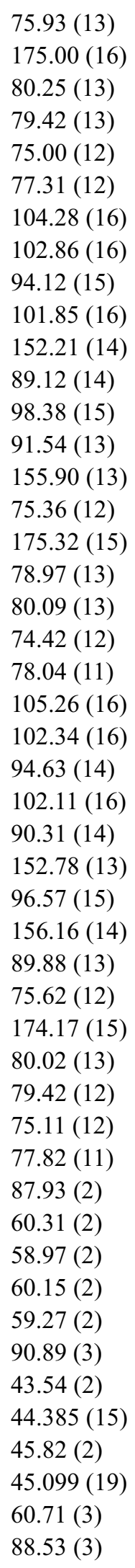

\begin{tabular}{|c|c|}
\hline $\mathrm{O} 9 \mathrm{~B}-\mathrm{K} 5-\mathrm{O} 22 \mathrm{~T}^{\mathrm{vii}}$ & $72.81(10)$ \\
\hline $\mathrm{O} 7 \mathrm{~W}-\mathrm{K} 5-\mathrm{O} 22 \mathrm{~T}^{\mathrm{vii}}$ & $76.63(12)$ \\
\hline $\mathrm{O} 8 \mathrm{~W}-\mathrm{K} 5-\mathrm{O} 9 \mathrm{~W}$ & $142.73(18)$ \\
\hline $\mathrm{O} 18 \mathrm{~B}^{\mathrm{vi}}-\mathrm{K} 5-\mathrm{O} 9 \mathrm{~W}$ & $100.56(14)$ \\
\hline $\mathrm{O} 9 \mathrm{~B}-\mathrm{K} 5-\mathrm{O} 9 \mathrm{~W}$ & $73.02(12)$ \\
\hline $\mathrm{O} 7 \mathrm{~W}-\mathrm{K} 5-\mathrm{O} 9 \mathrm{~W}$ & $92.42(15)$ \\
\hline $\mathrm{O} 22 \mathrm{~T}^{\mathrm{vii}-\mathrm{K} 5-\mathrm{O} 9 \mathrm{~W}}$ & $75.98(14)$ \\
\hline $\mathrm{O} 8 \mathrm{~W}-\mathrm{K} 5-\mathrm{O} 23 \mathrm{~T}$ & $78.72(14)$ \\
\hline $\mathrm{O} 18 \mathrm{~B}^{\mathrm{vi}}-\mathrm{K} 5-\mathrm{O} 23 \mathrm{~T}$ & $71.86(10)$ \\
\hline $\mathrm{O} 9 \mathrm{~B}-\mathrm{K} 5-\mathrm{O} 23 \mathrm{~T}$ & $55.51(10)$ \\
\hline $\mathrm{O} 7 \mathrm{~W}-\mathrm{K} 5-\mathrm{O} 23 \mathrm{~T}$ & $152.01(14)$ \\
\hline $\mathrm{O} 22 \mathrm{~T}^{\mathrm{vii}-\mathrm{K} 5-\mathrm{O} 23 \mathrm{~T}}$ & $117.87(11)$ \\
\hline $\mathrm{O} 9 \mathrm{~W}-\mathrm{K} 5-\mathrm{O} 23 \mathrm{~T}$ & $113.75(13)$ \\
\hline $\mathrm{Pt} 1-\mathrm{O} 1 \mathrm{D}-\mathrm{V} 4$ & 99.17 (13) \\
\hline Pt1-O1D-V9 & $94.73(12)$ \\
\hline $\mathrm{V} 4-\mathrm{O} 1 \mathrm{D}-\mathrm{V} 9$ & $92.37(12)$ \\
\hline $\mathrm{Pt} 1-\mathrm{O} 1 \mathrm{D}-\mathrm{V} 6$ & $93.70(12)$ \\
\hline $\mathrm{V} 4-\mathrm{O} 1 \mathrm{D}-\mathrm{V} 6$ & $93.47(12)$ \\
\hline $\mathrm{V} 9-\mathrm{O} 1 \mathrm{D}-\mathrm{V} 6$ & $168.86(15)$ \\
\hline $\mathrm{Pt} 1-\mathrm{O} 1 \mathrm{D}-\mathrm{V} 1$ & $92.47(12)$ \\
\hline $\mathrm{V} 4-\mathrm{O} 1 \mathrm{D}-\mathrm{V} 1$ & $168.36(15)$ \\
\hline $\mathrm{V} 9-\mathrm{O} 1 \mathrm{D}-\mathrm{V} 1$ & $86.35(11)$ \\
\hline $\mathrm{V} 6-\mathrm{O} 1 \mathrm{D}-\mathrm{V} 1$ & $86.01(10)$ \\
\hline $\mathrm{Pt} 1-\mathrm{O} 1 \mathrm{D}-\mathrm{V} 5$ & $174.40(16)$ \\
\hline $\mathrm{V} 4-\mathrm{O} 1 \mathrm{D}-\mathrm{V} 5$ & $86.40(11)$ \\
\hline $\mathrm{V} 9-\mathrm{O} 1 \mathrm{D}-\mathrm{V} 5$ & $85.49(10)$ \\
\hline $\mathrm{V} 6-\mathrm{O} 1 \mathrm{D}-\mathrm{V} 5$ & $85.42(10)$ \\
\hline $\mathrm{V} 1-\mathrm{O} 1 \mathrm{D}-\mathrm{V} 5$ & $81.96(10)$ \\
\hline $\mathrm{Pt} 1-\mathrm{O} 2 \mathrm{D}-\mathrm{V} 4$ & $99.47(13)$ \\
\hline $\mathrm{Pt} 1-\mathrm{O} 2 \mathrm{D}-\mathrm{V} 7$ & $95.20(12)$ \\
\hline $\mathrm{V} 4-\mathrm{O} 2 \mathrm{D}-\mathrm{V} 7$ & $93.10(11)$ \\
\hline $\mathrm{Pt} 1-\mathrm{O} 2 \mathrm{D}-\mathrm{V} 8$ & $94.04(12)$ \\
\hline $\mathrm{V} 4-\mathrm{O} 2 \mathrm{D}-\mathrm{V} 8$ & $93.16(12)$ \\
\hline $\mathrm{V} 7-\mathrm{O} 2 \mathrm{D}-\mathrm{V} 8$ & $167.84(15)$ \\
\hline $\mathrm{Pt} 1-\mathrm{O} 2 \mathrm{D}-\mathrm{V} 2$ & $92.35(11)$ \\
\hline $\mathrm{V} 4-\mathrm{O} 2 \mathrm{D}-\mathrm{V} 2$ & $168.17(15)$ \\
\hline $\mathrm{V} 7-\mathrm{O} 2 \mathrm{D}-\mathrm{V} 2$ & $85.63(11)$ \\
\hline $\mathrm{V} 8-\mathrm{O} 2 \mathrm{D}-\mathrm{V} 2$ & $86.10(10)$ \\
\hline $\mathrm{Pt} 1-\mathrm{O} 2 \mathrm{D}-\mathrm{V} 3$ & $173.86(16)$ \\
\hline $\mathrm{V} 4-\mathrm{O} 2 \mathrm{D}-\mathrm{V} 3$ & $86.57(10)$ \\
\hline $\mathrm{V} 7-\mathrm{O} 2 \mathrm{D}-\mathrm{V} 3$ & $85.52(10)$ \\
\hline $\mathrm{V} 8-\mathrm{O} 2 \mathrm{D}-\mathrm{V} 3$ & $84.47(10)$ \\
\hline $\mathrm{V} 2-\mathrm{O} 2 \mathrm{D}-\mathrm{V} 3$ & $81.61(10)$ \\
\hline $\mathrm{V} 6-\mathrm{O} 3 \mathrm{C}-\mathrm{Pt} 1$ & $101.52(13)$ \\
\hline $\mathrm{V} 6-\mathrm{O} 3 \mathrm{C}-\mathrm{V} 7$ & $99.96(13)$ \\
\hline $\mathrm{Pt} 1-\mathrm{O} 3 \mathrm{C}-\mathrm{V} 7$ & $101.69(14)$ \\
\hline $\mathrm{Pt} 1-\mathrm{O} 4 \mathrm{C}-\mathrm{V} 8$ & $100.70(13)$ \\
\hline $\mathrm{Pt} 1-\mathrm{O} 4 \mathrm{C}-\mathrm{V} 9$ & $100.45(13)$ \\
\hline
\end{tabular}




\begin{tabular}{|c|c|c|c|}
\hline $\mathrm{V} 7-\mathrm{V} 2-\mathrm{Pt} 1$ & $59.81(2)$ & $\mathrm{V} 8-\mathrm{O} 4 \mathrm{C}-\mathrm{V} 9$ & $100.37(13)$ \\
\hline $\mathrm{V} 3-\mathrm{V} 2-\mathrm{V} 8$ & $59.96(3)$ & $\mathrm{V} 4-\mathrm{O} 5 \mathrm{C}-\mathrm{V} 7$ & $109.01(15)$ \\
\hline $\mathrm{V} 7-\mathrm{V} 2-\mathrm{V} 8$ & $91.11(3)$ & $\mathrm{V} 4-\mathrm{O} 5 \mathrm{C}-\mathrm{V} 6$ & $109.70(15)$ \\
\hline $\mathrm{Pt} 1-\mathrm{V} 2-\mathrm{V} 8$ & $59.15(2)$ & $\mathrm{V} 7-\mathrm{O} 5 \mathrm{C}-\mathrm{V} 6$ & $101.51(14)$ \\
\hline $\mathrm{V} 3-\mathrm{V} 2-\mathrm{V} 4$ & $43.98(2)$ & $\mathrm{V} 4-\mathrm{O} 6 \mathrm{C}-\mathrm{V} 9$ & 109.15 (14) \\
\hline $\mathrm{V} 7-\mathrm{V} 2-\mathrm{V} 4$ & $45.49(2)$ & $\mathrm{V} 4-\mathrm{O} 6 \mathrm{C}-\mathrm{V} 8$ & $108.85(15)$ \\
\hline $\mathrm{Pt} 1-\mathrm{V} 2-\mathrm{V} 4$ & $44.562(14)$ & $\mathrm{V} 9-\mathrm{O} 6 \mathrm{C}-\mathrm{V} 8$ & $103.00(13)$ \\
\hline $\mathrm{V} 8-\mathrm{V} 2-\mathrm{V} 4$ & 45.641 (19) & $\mathrm{Pt} 1-\mathrm{O} 7 \mathrm{~B}-\mathrm{V} 2$ & $100.62(13)$ \\
\hline $\mathrm{V} 3-\mathrm{V} 2-\mathrm{V} 1$ & $89.54(2)$ & $\mathrm{Pt} 1-\mathrm{O} 8 \mathrm{~B}-\mathrm{V} 1$ & $101.22(14)$ \\
\hline $\mathrm{V} 7-\mathrm{V} 2-\mathrm{V} 1$ & $59.98(2)$ & $\mathrm{V} 4-\mathrm{O} 10 \mathrm{~B}-\mathrm{V} 3$ & $112.76(17)$ \\
\hline $\mathrm{Pt} 1-\mathrm{V} 2-\mathrm{V} 1$ & $1.113(10)$ & $\mathrm{V} 4-\mathrm{O} 11 \mathrm{~B}-\mathrm{V} 5$ & $112.09(16)$ \\
\hline $\mathrm{V} 8-\mathrm{V} 2-\mathrm{V} 1$ & $60.11(2)$ & $\mathrm{V} 5-\mathrm{O} 12 \mathrm{~B}-\mathrm{V} 1$ & $116.48(17)$ \\
\hline $\mathrm{V} 4-\mathrm{V} 2-\mathrm{V} 1$ & $45.564(14)$ & $\mathrm{V} 6-\mathrm{O} 13 \mathrm{~B}-\mathrm{V} 1$ & $119.14(17)$ \\
\hline $\mathrm{V} 2-\mathrm{V} 3-\mathrm{V} 4$ & $92.11(3)$ & $\mathrm{V} 6-\mathrm{O} 14 \mathrm{~B}-\mathrm{V} 5$ & $117.51(16)$ \\
\hline $\mathrm{V} 2-\mathrm{V} 3-\mathrm{V} 8$ & $60.89(3)$ & $\mathrm{V} 7-\mathrm{O} 15 \mathrm{~B}-\mathrm{V} 3$ & $117.70(17)$ \\
\hline $\mathrm{V} 4-\mathrm{V} 3-\mathrm{V} 8$ & $61.69(3)$ & $\mathrm{V} 7-\mathrm{O} 16 \mathrm{~B}-\mathrm{V} 2$ & $117.32(17)$ \\
\hline $\mathrm{V} 2-\mathrm{V} 3-\mathrm{V} 7$ & $60.19(3)$ & $\mathrm{V} 8-\mathrm{O} 17 \mathrm{~B}-\mathrm{V} 2$ & $118.96(16)$ \\
\hline $\mathrm{V} 4-\mathrm{V} 3-\mathrm{V} 7$ & $61.23(2)$ & $\mathrm{V} 8-\mathrm{O} 18 \mathrm{~B}-\mathrm{V} 3$ & $117.66(17)$ \\
\hline $\mathrm{V} 8-\mathrm{V} 3-\mathrm{V} 7$ & $91.34(3)$ & $\mathrm{V} 9-\mathrm{O} 19 \mathrm{~B}-\mathrm{V} 1$ & $116.70(16)$ \\
\hline $\mathrm{V} 5-\mathrm{V} 4-\mathrm{Pt} 1$ & $88.17(2)$ & $\mathrm{V} 9-\mathrm{O} 20 \mathrm{~B}-\mathrm{V} 5$ & 117.45 (17) \\
\hline $\mathrm{V} 3-\mathrm{V} 4-\mathrm{Pt} 1$ & $88.48(2)$ & $\mathrm{H} 1 \mathrm{~A}-\mathrm{O} 1 \mathrm{~W}-\mathrm{H} 1 \mathrm{~B}$ & $109(5)$ \\
\hline $\mathrm{V} 5-\mathrm{V} 4-\mathrm{V} 9$ & $60.06(2)$ & $\mathrm{H} 2 \mathrm{~A}-\mathrm{O} 2 \mathrm{~W}-\mathrm{H} 2 \mathrm{~B}$ & $102(4)$ \\
\hline $\mathrm{V} 3-\mathrm{V} 4-\mathrm{V} 9$ & $118.47(3)$ & $\mathrm{H} 3 \mathrm{~A}-\mathrm{O} 3 \mathrm{~W}-\mathrm{H} 3 \mathrm{~B}$ & $107(5)$ \\
\hline Pt1-V4-V9 & 59.155 (19) & $\mathrm{H} 4 \mathrm{~A}-\mathrm{O} 4 \mathrm{~W}-\mathrm{H} 4 \mathrm{~B}$ & $109(4)$ \\
\hline $\mathrm{V} 5-\mathrm{V} 4-\mathrm{V} 7$ & $117.42(3)$ & $\mathrm{H} 5 \mathrm{~A}-\mathrm{O} 5 \mathrm{~W}-\mathrm{H} 5 \mathrm{~B}$ & $106(4)$ \\
\hline $\mathrm{V} 3-\mathrm{V} 4-\mathrm{V} 7$ & $60.12(3)$ & $\mathrm{H} 6 \mathrm{~A}-\mathrm{O} 6 \mathrm{~W}-\mathrm{H} 6 \mathrm{~B}$ & 109.4 \\
\hline Pt1-V4-V7 & $59.28(2)$ & $\mathrm{H} 7 \mathrm{~A}-\mathrm{O} 7 \mathrm{~W}-\mathrm{H} 7 \mathrm{~B}$ & $105(4)$ \\
\hline $\mathrm{V} 9-\mathrm{V} 4-\mathrm{V} 7$ & $118.44(3)$ & $\mathrm{H} 8 \mathrm{~A}-\mathrm{O} 8 \mathrm{~W}-\mathrm{H} 8 \mathrm{~B}$ & $103(4)$ \\
\hline $\mathrm{V} 5-\mathrm{V} 4-\mathrm{V} 8$ & $118.99(3)$ & $\mathrm{H} 9 \mathrm{~A}-\mathrm{O} 9 \mathrm{~W}-\mathrm{H} 9 \mathrm{~B}$ & $101(4)$ \\
\hline $\mathrm{V} 3-\mathrm{V} 4-\mathrm{V} 8$ & $59.49(2)$ & & \\
\hline
\end{tabular}

Symmetry codes: (i) $x, y+1, z$; (ii) $x, y, z+1$; (iii) $x, y+1, z+1$; (iv) $-x,-y+2,-z+1$; (v) $-x+1,-y+2,-z+1$; (vi) $-x+1,-y+1,-z+1$; (vii) $-x,-y+1,-z+1$; (viii) $x, y-1, z ;$ (ix) $-x,-y+1,-z$.

Hydrogen-bond geometry $\left(\AA,{ }^{\circ}\right)$

\begin{tabular}{lllll}
\hline$D-\mathrm{H} \cdots A$ & $D-\mathrm{H}$ & $\mathrm{H} \cdots A$ & $D \cdots A$ & $D-\mathrm{H} \cdots A$ \\
\hline $\mathrm{O} 7 B-\mathrm{H} 7 \cdots \mathrm{O} 19 B^{\text {ix }}$ & $0.90(8)$ & $1.86(8)$ & $2.738(4)$ & $164(8)$ \\
$\mathrm{O} 8 B-\mathrm{H} 8 \cdots \mathrm{O} 4 C^{\text {ix }}$ & $0.77(6)$ & $1.90(6)$ & $2.645(5)$ & $161(6)$ \\
$\mathrm{O} 1 W-\mathrm{H} 1 B \cdots \mathrm{O} 9 B$ & $0.83(3)$ & $2.50(6)$ & $3.127(6)$ & $133(7)$ \\
$\mathrm{O} 1 W-\mathrm{H} 1 A \cdots \mathrm{O} 8 W$ & $0.84(3)$ & $2.10(3)$ & $2.930(9)$ & $170(8)$ \\
$\mathrm{O} 2 W-\mathrm{H} 2 A \cdots \mathrm{O} 15 B$ & $0.87(3)$ & $1.88(3)$ & $2.728(6)$ & $166(8)$ \\
$\mathrm{O} 2 W-\mathrm{H} 2 B \cdots \mathrm{O} 11 B^{\mathrm{v}}$ & $0.86(3)$ & $2.23(5)$ & $2.975(6)$ & $145(8)$ \\
$\mathrm{O} 3 W-\mathrm{H} 3 A \cdots \mathrm{O} 3 C^{\text {vii }}$ & $0.84(3)$ & $1.83(3)$ & $2.673(5)$ & $177(10)$ \\
$\mathrm{O} 3 W-\mathrm{H} 3 B \cdots \mathrm{O} 7 W$ & $0.83(3)$ & $2.09(5)$ & $2.875(7)$ & $158(8)$ \\
$\mathrm{O} 4 W-\mathrm{H} 4 A \cdots \mathrm{O} 13 B^{\text {ix }}$ & $0.83(3)$ & $1.88(3)$ & $2.680(5)$ & $162(7)$ \\
$\mathrm{O} 4 W-\mathrm{H} 4 B \cdots \mathrm{O} 14 B^{\text {viii }}$ & $0.83(3)$ & $2.10(4)$ & $2.850(5)$ & $151(7)$ \\
$\mathrm{O} 5 W-\mathrm{H} 5 A \cdots \mathrm{O} 2 W^{\text {ii }}$ & $0.87(3)$ & $1.84(3)$ & $2.710(8)$ & $175(8)$ \\
$\mathrm{O} 5 W-\mathrm{H} 5 B \cdots \mathrm{O} 24 T^{\mathrm{x}}$ & $0.84(3)$ & $2.26(5)$ & $2.972(6)$ & $142(7)$
\end{tabular}


supporting information

$\begin{array}{lllll}\mathrm{O} 6 W-\mathrm{H} 6 A \cdots \mathrm{O} 5 C^{\mathrm{vii}} & 0.97 & 1.81 & 2.755(5) & 163 \\ \mathrm{O} 6 W-\mathrm{H} 6 B \cdots \mathrm{O} 10 B^{\mathrm{vi}} & 0.97 & 2.04 & 2.755(5) & 129 \\ \mathrm{O} 7 W-\mathrm{H} 7 A \cdots \mathrm{O} 7 B^{\mathrm{vii}} & 0.85(3) & 2.07(4) & 2.891(5) & 163(8) \\ \mathrm{O} 7 W-\mathrm{H} 7 B \cdots \mathrm{O} 6 C^{\mathrm{vi}} & 0.84(3) & 2.27(6) & 2.885(5) & 130(6) \\ \mathrm{O} 8 W-\mathrm{H} 8 A \cdots \mathrm{O} 5 W^{\mathrm{vi}} & 0.86(3) & 1.98(5) & 2.795(7) & 159(10) \\ \mathrm{O} 8 W-\mathrm{H} 8 B \cdots \mathrm{O} 19 B^{\mathrm{ii}} & 0.86(3) & 2.22(4) & 3.031(6) & 156(8)\end{array}$

Symmetry codes: (ii) $x, y, z+1$; (v) $-x+1,-y+2,-z+1$; (vi) $-x+1,-y+1,-z+1$; (vii) $-x,-y+1,-z+1$; (viii) $x, y-1, z$; (ix) $-x,-y+1,-z$; (x) $-x+1,-y+1,-z$. 